\title{
Article \\ One- and Two-Phase Software Requirement Classification Using Ensemble Deep Learning
}

\author{
Nouf Rahimi ${ }^{1,2, *(\mathbb{D})}$, Fathy Eassa ${ }^{1}$ and Lamiaa Elrefaei $^{3}(\mathbb{D}$ \\ 1 Computer Science Department, Faculty of Computing and Information Technology, King Abdul-Aziz \\ University, Jeddah 21589, Saudi Arabia; feassa@kau.edu.sa \\ 2 Information System and Technology Department, Faculty of Computer Science and Engineering, University \\ of Jeddah, Jeddah 21959, Saudi Arabia \\ 3 Electrical Engineering Department, Faculty of Engineering at Shoubra, Benha University, Cairo 11629, Egypt; \\ lamia.alrefaai@feng.bu.edu.eg \\ * Correspondence: rahiminouf@gmail.com; Tel.: +966-533535011
}

Citation: Rahimi, N.; Eassa, F.;

Elrefaei, L. One- and Two-Phase Software Requirement Classification Using Ensemble Deep Learning. Entropy 2021, 23, 1264. https:// doi.org/10.3390/e23101264

Academic Editor: Gholamreza Anbarjafari

Received: 8 August 2021

Accepted: 27 September 2021

Published: 28 September 2021

Publisher's Note: MDPI stays neutral with regard to jurisdictional claims in published maps and institutional affiliations.

Copyright: (c) 2021 by the authors. Licensee MDPI, Basel, Switzerland. This article is an open access article distributed under the terms and conditions of the Creative Commons Attribution (CC BY) license (https:// creativecommons.org/licenses/by/ $4.0 /)$.

\begin{abstract}
Recently, deep learning (DL) has been utilized successfully in different fields, achieving remarkable results. Thus, there is a noticeable focus on DL approaches to automate software engineering (SE) tasks such as maintenance, requirement extraction, and classification. An advanced utilization of DL is the ensemble approach, which aims to reduce error rates and learning time and improve performance. In this research, three ensemble approaches were applied: accuracy as a weight ensemble, mean ensemble, and accuracy per class as a weight ensemble with a combination of four different DL models-long short-term memory (LSTM), bidirectional long short-term memory (BiLSTM), a gated recurrent unit (GRU), and a convolutional neural network (CNN) - in order to classify the software requirement (SR) specification, the binary classification of SRs into functional requirement (FRs) or non-functional requirements (NFRs), and the multi-label classification of both FRs and NFRs into further experimental classes. The models were trained and tested on the PROMISE dataset. A one-phase classification system was developed to classify SRs directly into one of the 17 multi-classes of FRs and NFRs. In addition, a two-phase classification system was developed to classify SRs first into FRs or NFRs and to pass the output to the second phase of multi-class classification to 17 classes. The experimental results demonstrated that the proposed classification systems can lead to a competitive classification performance compared to the state-of-the-art methods. The two-phase classification system proved its robustness against the one-phase classification system, as it obtained a $95.7 \%$ accuracy in the binary classification phase and a $93.4 \%$ accuracy in the second phase of NFR and FR multi-class classification.
\end{abstract}

Keywords: software requirement; functional requirement; non-functional requirement; classification; deep learning; ensemble; CNN; GRU; BiLSTM; LSTM

\section{Introduction}

Software requirement (SR) classification is carried out by software specialists to directly determine the requirements that they need or are interested in. For example, user interface designers are probably interested in "look and feel" requirements. The manual labeling or classification of each requirement to determine which category it belongs to is expensive and requires experts in different fields, which results in high costs. This provides motivation for finding a complete system with high performance to label and classify SR into functional requirements (FRs) or non-functional requirement (NFRs), as well as both FRs and NFRs into further classes [1].

The main classes of SRs are FRs and NFRs. FRs are defined according to the IEEE Standard Glossary of Software Engineering Terminology as a function carried out by a system or its components [2]. On the contrary, NFRs are one of the restrictions on system 
behavior [3]. Additionally, there are subcategories of both FRs and NFRs, as shown in Table 1 (FR subcategories [2]) and Table 2 (NFR subcategories [4]).

Table 1. Six different classes of functional requirements (FRs) [4].

\begin{tabular}{|c|c|c|}
\hline Class & Definition & Example \\
\hline Solution & $\begin{array}{l}\text { Includes actions that are expected to be } \\
\text { carried out by the system or different users. }\end{array}$ & $\begin{array}{c}\text { "The system shall display completed worklist items to the Lab } \\
\text { Manager." }\end{array}$ \\
\hline Enablement & $\begin{array}{l}\text { Includes the capabilities offered by the } \\
\text { system to different users. Subsystems that } \\
\text { offer these capabilities are optionally } \\
\text { specified. }\end{array}$ & "The Lab Manager shall be able to create worklist items." \\
\hline $\begin{array}{l}\text { Attribute } \\
\text { Constraint }\end{array}$ & $\begin{array}{l}\text { Constraints on attributes or entity } \\
\text { attributes are specified through this class. }\end{array}$ & $\begin{array}{l}\text { "Search options must always be one of the following: Price, } \\
\text { destination, restaurant type, or specific dish." }\end{array}$ \\
\hline $\begin{array}{l}\text { Action } \\
\text { Constraint }\end{array}$ & $\begin{array}{l}\text { Allowed and non-allowed actions by the } \\
\text { system or the subsystems displayed using } \\
\text { this class. }\end{array}$ & $\begin{array}{c}\text { "The loan subsystem may only delete a lender if there are no loans } \\
\text { in the portfolio associated with this lender." }\end{array}$ \\
\hline Policy & $\begin{array}{l}\text { This class is responsible for specifying all } \\
\text { policies that have been decided for the } \\
\text { system. }\end{array}$ & "A loan is not computed in more than one bundle." \\
\hline Definition & All entities are defined using this class. & $\begin{array}{l}\text { "The expected profit of a fixed-rate loan is defined as the amount of } \\
\text { interest received over the remaining life of the loan." }\end{array}$ \\
\hline
\end{tabular}

Table 2. Non-functional requirement (NFR) subcategories [5].

\begin{tabular}{|c|c|c|}
\hline Class & Definition & Example \\
\hline Availability & $\begin{array}{l}\text { Related to users' accessibility to a system at } \\
\text { a specific time. }\end{array}$ & $\begin{array}{l}\text { "The product shall be available } 24 \mathrm{~h} \text { per day, seven days per } \\
\text { week." }\end{array}$ \\
\hline Fault Tolerance & $\begin{array}{l}\text { Determines the degree to which the system } \\
\text { or product operates in the absence of } \\
\text { hardware or software faults. }\end{array}$ & $\begin{array}{l}\text { "100\% of saved user preferences shall be restored when the } \\
\text { system comes back online." }\end{array}$ \\
\hline $\begin{array}{l}\text { Legal and } \\
\text { Licensing }\end{array}$ & $\begin{array}{l}\text { Determines the licenses and certificates that } \\
\text { the system needs to obtain. }\end{array}$ & $\begin{array}{l}\text { "All actions that modify an existing dispute case must be } \\
\text { recorded in the case history." }\end{array}$ \\
\hline Look and Feel & Specifies the appearance and style. & "The website design should be modern, clean, and concise." \\
\hline Maintainability & $\begin{array}{l}\text { Effectiveness and efficiency of a system } \\
\text { modified by maintainers. }\end{array}$ & $\begin{array}{c}\text { "Application updates shall occur between 3:00 a.m. and 6:00 a.m. } \\
\text { CST on Wednesday morning during the middle of the NFL } \\
\text { season." }\end{array}$ \\
\hline Operability & $\begin{array}{l}\text { How easy to operate and control a system } \\
\text { through its attributes. }\end{array}$ & $\begin{array}{c}\text { "The product shall run on the existing hardware for all } \\
\text { environments." }\end{array}$ \\
\hline Performance & $\begin{array}{l}\text { Related to required resources at a specific } \\
\text { condition. }\end{array}$ & $\begin{array}{l}\text { "A customer shall be able to check the status of their prepaid card } \\
\text { by entering in the PIN number in under } 5 \text { s." }\end{array}$ \\
\hline Portability & $\begin{array}{l}\text { The effectiveness and efficiency in } \\
\text { transferring the system from one hardware, } \\
\text { software, or environment to another. }\end{array}$ & $\begin{array}{c}\text { "The product is expected to run on Windows CE and Palm } \\
\text { operating systems." }\end{array}$ \\
\hline Scalability & $\begin{array}{l}\text { The degree to which a system adapts } \\
\text { effectively and efficiently from one } \\
\text { hardware, software, or environment to } \\
\text { another. }\end{array}$ & $\begin{array}{c}\text { "The system shall be able to handle all of the user requests/usage } \\
\text { during business hours." }\end{array}$ \\
\hline Security & $\begin{array}{l}\text { Includes the protection of personal data } \\
\text { and authorized access. }\end{array}$ & "The product shall provide authentication and authorization." \\
\hline Usability & $\begin{array}{l}\text { The level of efficiency and effectiveness of a } \\
\text { system that can be used by specific users to } \\
\text { reach specific goals with satisfaction. }\end{array}$ & $\begin{array}{l}\text { "The product shall be installed by an untrained realtor without } \\
\text { recourse to separately printed instructions." }\end{array}$ \\
\hline
\end{tabular}


Deep learning (DL) has been adopted to automate and facilitate the tasks of software engineering (SE), such as maintenance, requirement extraction, and classification [6]. The results have been interesting, but some areas have not been extensively covered. SR classification plays a crucial role in software development as it facilitates the process and helps in avoiding further modification costs [7]. Moreover, it is not the matter of classification but the correct classification of SRs that is the crucial task in SE [3]. The correct classification of SRs into FRs or NFRs and the subcategories of NFRs and FRs are described as challenges due to many reasons related to the inconsistency among the different stakeholders and engineers, as they use different structures for the different classes, and the terms are not unified either. Thus, such automation of this classification is greatly needed [3].

Automation of the SR classification has been the main objective of a number of previous studies. Some of these studies used traditional automation tools and implemented simple software and tools, but there were disadvantages such as the complexity of the tools, incomplete extraction and classification (as NFRs were the main concern for the majority), unavailability, and low accuracy. ML or DL classifiers have been used in recent studies for SR classification as well. However, weaknesses have been observed among ML and DL solutions, such as incomplete solutions (as FRs have rarely been classified or ignored in some studies), accuracy issues, and availability problems. To overcome the weaknesses and the disadvantages of existing solutions, this study aimed to automate SR classification using DL models, along with ensemble methods, to form a complete model that conducted binary and multi-class classification for both FRs and NFRs.

The ensemble method is used more often than the DL method, although both are assumed to be good in different ways and do not make similar prediction errors. However, the results of the ensemble method are less sensitive to the specifications of training data. Moreover, it has been confirmed that the performance of a group of models is better than that of a single model [8]. Thus, ensemble learning was used in this research, mainly because it is an approved method to enhance the accuracy in a variety of scientific fields.

The use of ensemble models for SR classification is rare, especially in the field. In this work, different models were applied and the performance of the existing models and a new recent ensemble model were evaluated for first time using DL models, with accuracy per class as a weight. The results indicated its robustness in all experiments, achieving the best accuracy. Complete SR classification is also provided using one- and two-phase systems.

The contributions of this paper include:

1. Summary, categorization, and comparison of published research on ML or DL use for classifying SRs. The summarized categories involve classifying SRs into main classes, classifying FRs into multi-classes, classifying NFRs into multi-classes, and complete systems that classify SRs into main classes and FRs and NFRs into multi-classes.

2. Introduction of an ensemble learning framework based on DL models for classifying different types of SRs. The conducted experiments present comparative results between three different ensemble models-mean ensemble, accuracy as a weight ensemble, and accuracy per class as a weight ensemble-and show that accuracy per class as a weight ensemble learning classifier combining BiLSTM, LSTM, GRU, and CNN has a stronger capability to correctly predict different types of SRs.

3. Proposition of a two-phase system using the ensemble DL method. The first phase uses binary classification to classify SRs into FRs or NFRs, while the second phase classifies the output of the first phase (FRs or NFRs) into multi-classes. FRs are classified into six different labels: solution, enablement, action constraint, attribute constraint, policy, and definition. On the contrary, NFRs are classified into 11 different labels: availability, fault tolerance, legal and licensing, look and feel, maintainability, operability, performance, portability, scalability, security, and usability.

4. Proposition of a one-phase system using the ensemble DL method. The input is SRs and the output is 17 multi-classes of either FRs or NFRs (solution, enablement, action constraint, attribute constraint, policy, definition, availability, fault tolerance, legal 
and licensing, look and feel, maintainability, operability, performance, portability, scalability, security, and usability).

5. Investigation of the performance of each base DL classifier and a number of ensemble DL classifiers in each phase of the system.

6. To the best of our knowledge there is no complete two-phase system to classify SRs into FRs or NFRs, then to classify each FR and NFR into multi-classes using ensemble DL methodology. In addition, there is no such similar categorized review summary of the previous studies on classifying SRs.

The rest of the paper is organized as follows: Section 2 presents a review of the state of automated SR classification using the ML and DL methods. Section 3 displays the used methodology in detail, while Section 4 explains the details of the conducted experiments. Section 5 provides a comparative analysis, and Section 6 concludes this research.

\section{Related Work}

This section summarizes previous work regarding the ML and DL methods that try to automate the classification of SRs into different categories: the binary classification of SRs into FRs or NFRs (Section 2.1), the multi-class classification of FRs (Section 2.2), the multi-class classification of NFRs (Section 2.3), and the complete systems that classify SRs into FRs or NFRs and both FRs and NFRs into subcategories (Section 2.4).

\subsection{Classifying SRs into Main Classes}

In [9], the authors assigned software requirements to multiple topics. The support vector machine (SVM) and multinomial naïve Bayes (MNB) models were used for classification purposes. Two German specifications by Mercedes-Benz were used, one of which was public and the other a confidential dataset. The results of these experiments recorded a maximum recall of 0.94 by the $\mathrm{MNB}$ classifier and a maximum precision of 0.86 by the SVM classifier.

In [10], a convolutional neural network (CNN) was used to classify text as either a software requirement or information. The Data Object Oriented Repository System (DOORS) dataset document, which was collected from 89 requirement specifications, was used to train and test the model. Using the $\mathrm{CNN}$ model to achieve the desired goal of classification recorded an accuracy of $81 \%$, a precision of 0.73 , and a recall of 0.89 .

\subsection{Classifying FRs into Multi-Classes}

The ensemble ML approach introduced in [11] aimed to classify FRs into six categories: Solution, enablement, action constraint, attribute constraint, definition, and policy. The ensemble method was an enhancement of accuracy as weight, as it used the accuracy per class as a weight to find the best classifier for each class. The ensemble combined five ML classifiers: SVM, naïve Bayes, logistic regression (LR), support vector classification (SVC), and decision tree. The model was trained and tested on a collected dataset that contained 600 FR statements with the same number of FRs from each class. The use of the most accurate three classifiers (SVM, SVC, and LR) outperformed the technique using all classifiers and achieved a $99.45 \%$ accuracy in $0.7 \mathrm{~s}$.

\subsection{Classifying NFRs into Multi-Classes}

In [12], NFRs were categorized into approximately 14 categories, such as capacity, reliability, and security. The model was based on the K-nearest neighbor classifier and was tested on 11 different documents related to electronic health records (EHRs). The classifier was compared to the naïve Bayes and support vector machine classifiers and scored higher results with an F1 score of approximately 0.54 .

In [13], NFRs were categorized into different categories: Availability, look and feel, legal, maintainability, operational, performance, scalability, security, and usability. However, FRs were not categorized into deeper categories, and any software requirement that did not belong to any category was classified as an FR. The proposed classifier was designed 
to classify based on the frequency of terms that belonged to each category and calculated their weight using term frequency-inverse document frequency (TF-IDF). The classifier was trained and tested on 15 project documents that included 326 NFRs and 358 FRs. The results showed that considering only the top 15 frequent terms was the best technique, with a recall of 0.7669 .

In [14], the authors used the same dataset as that used in [13] but a different methodology. The naïve Bayes classifier was used, trained, and tested on more than 600 instances. To boost the classification, the expectation maximization algorithm was used to label untrained data. The overall accuracy was approximately $97 \%$.

In [15], the authors conducted a number of experiments to compare the classification of FRs and NFRs using an ensemble that combined two ML techniques: Random forest and gradient boosting. Raw data were in the of SQL and CSV formats. NFRs were classified into approximately 10 labels such as security, performance, and usability. FRs were not classified into further classes. The experiments aimed to measure the accuracy of FR and NFR classification. The results showed that for classifying NFRs, the gradient boosting algorithm performed better than random forest $(0.826)$, while random forest was better at classifying FRs (0.591).

In [16], SRs were categorized as FRs or NFRs using the naïve Bayes classifier. Then, NFRs were further categorized into classes such as security, availability, and scalability. The collected data for the experiment by the authors included 255 FRs, and the number of NFRs ranged from 10 to 67 per category. The classifier scored a $75 \%$ precision for NFRs.

In [17], the PROMISE software engineering repository dataset was used to compare the performance of five ML algorithms (multinomial naïve Bayes (MNB), Gaussian naïve Bayes (GNB), Bernoulli naïve Bayes (BNB), K-nearest neighbor (KNN), support vector machine (SVM), stochastic gradient descent SVM (SGD SVM), and decision tree (Dtree)), along with different feature extraction techniques. Eleven categories were used to label NFRs into the categories of availability, legal, look and feel, maintainability, operational, performance, scalability, security, usability, fault tolerance, and portability. SGD SVM with TF-IDF scored the highest results of all of the classifiers, with a precision of 0.66 , a recall of 0.61 , an F1 score of 0.61 , and an accuracy of 0.76 .

In [7], the authors also used $\mathrm{CNN}$ for classification purposes. The authors classified SRs into the categories of functional, availability, legal, look and feel, maintainability, operational, performance, scalability, security, usability, fault tolerance, and portability. The PROMISE corpus dataset was used for the experiment with the proposed DL model. The evaluation of the performance was measured using precession, recall, and F score; the results for these measures were $0.80,0.785$, and 0.77 , respectively.

In [18], a Master's thesis was presented, which conducted experiments to perform a number of classifications: The binary classification of SRs into FRs or NFRs, the binary classification of NFRs (security-related and non-security-related), and the multi-label classification of NFRs (availability, legal, maintainability, operational, performance, scalability, look and feel, security, and usability). The PROMISE dataset was used to train and test the proposed $\mathrm{CNN}$ model. The results showed that the $\mathrm{CNN}$ classifier scored recall values of $0.945,0.911$, and 0.772 for the binary option of FRs or NFRs, the bidirectional encoder representations from transformers binary option of being security-related or not, and for NFR multi-label classification, respectively.

In [19], the authors classified NFRs into multi-classes using a specific type of tree classifier trained and tested using the Certification Commission for Healthcare Information Technology (CCHIT) dataset, achieving 93.92\%.

\subsection{Complete System (Classifying NFRs and FRs into Multi-Classes)}

In [20], the authors experimented with different classifications: Classifying SRs into FRs or NFRs, classifying NFRs into different categories (usability, security, operational, and performance), and classifying FRs into the categories of functions, data, and behavior. The model was a tuned (BERT) model named "NoRBERT". Then, it was trained and tested 
on the PROMISE dataset for all experiments. The model recorded satisfactory F1 scores compared to existing state-of-the-art methods for the first classification objective $(90 \%$ for FRs and $93 \%$ for NFRs). In NFR classification, it scored F1 scores of $76 \%$ and $92 \%$ for FR classification.

Analysis of the existing research on SR classification using the ML and DL methods, as summarized in Table 3, shows that different classifiers have been utilized, such as SVM, naïve Bayes, and CNN. However, binary classification or NFR classification into subcategories has been the main concern of the majority of studies, while FRs have attracted less attention and have rarely been classified into further classes. In addition, a complete system that classifies both FRs and NFRs into multi-classes has rarely been introduced, providing the motivation to conduct this piece of work.

Table 3. Summary of related work.

\begin{tabular}{|c|c|c|c|c|c|c|c|}
\hline \multirow{2}{*}{$\begin{array}{c}\text { Reference \# } \\
\text { Year }\end{array}$} & \multicolumn{2}{|c|}{ Classification } & \multirow{2}{*}{ Methodology } & \multirow{2}{*}{ Dataset } & \multirow{2}{*}{ Results } & \multirow{2}{*}{ Advantages } & \multirow{2}{*}{ Disadvantages } \\
\hline & Input & Classes & & & & & \\
\hline \multicolumn{8}{|c|}{ 1. Classifying SRs into Main Classes } \\
\hline [9] 2013 & $\begin{array}{l}\text { Software } \\
\text { require- } \\
\text { ments } \\
\text { (SRs) }\end{array}$ & Topics & $\begin{array}{c}\text { Support Vector } \\
\text { Machine (SVM) and } \\
\text { Multinomial Naïve } \\
\text { Bayes (MNB) }\end{array}$ & $\begin{array}{l}\text { Two German } \\
\text { specifications by } \\
\text { Mercedes-Benz } \\
\text { (public and } \\
\text { confidential) }\end{array}$ & $\begin{array}{l}\text { Recall: } 0.94 \\
\text { (MNB) } \\
\text { Precision: } 0.86 \\
\text { (SVM) }\end{array}$ & $\begin{array}{l}\text { Reliable results. } \\
\text { Improves } \\
\text { technical } \\
\text { review to } \\
\text { enhance SRs. }\end{array}$ & $\begin{array}{l}\text { No enough } \\
\text { training data. }\end{array}$ \\
\hline [10] 2017 & Text & $\begin{array}{l}\text { Requirement } \\
\text { OR } \\
\text { Information }\end{array}$ & $\begin{array}{l}\text { Convolutional Neural } \\
\text { Network (CNN) }\end{array}$ & $\begin{array}{l}\text { DOORS document } \\
\text { database }\end{array}$ & $\begin{array}{l}\text { Accuracy: } 81 \% \\
\text { Precision: } 0.73 \\
\text { Recall: } 0.89\end{array}$ & $\begin{array}{l}\text { One of the first } \\
\text { studies that } \\
\text { used CNN. }\end{array}$ & $\begin{array}{l}\text { Does not point } \\
\text { to wrongly } \\
\text { classified } \\
\text { input. }\end{array}$ \\
\hline \multicolumn{8}{|c|}{ 2. Classifying FRs into Multi-Classes } \\
\hline [11] 2020 & $\begin{array}{l}\text { Functional } \\
\text { require- } \\
\text { ments } \\
\text { (FRs) }\end{array}$ & $\begin{array}{c}\text { Solution } \\
\text { Enablement } \\
\text { Action constraint } \\
\text { Attribute constraint } \\
\text { Definition } \\
\text { Policy }\end{array}$ & $\begin{array}{l}\text { Ensemble (accuracy } \\
\text { per class as a weight) } \\
\text { includes support } \\
\text { vector classification, } \\
\text { support vector } \\
\text { machine, decision } \\
\text { tree, logistic } \\
\text { regression, and naïve } \\
\text { Bayes }\end{array}$ & $\begin{array}{l}\text { Collected dataset that } \\
\text { has } 600 \text { FR statements } \\
\text { with } 100 \text { FRs from } \\
\text { each category }\end{array}$ & $\begin{array}{l}\text { Accuracy: } \\
99.45 \% \\
\text { Time: } 0.07 \text { s }\end{array}$ & $\begin{array}{c}\text { Novel ensemble } \\
\text { approach. } \\
\text { New } \\
\text { introduced FR } \\
\text { dataset. }\end{array}$ & $\begin{array}{l}\text { Limited to } \\
\text { FRs. }\end{array}$ \\
\hline \multicolumn{8}{|c|}{ 3. Classifying Non-Functional Requirements (NFRs) into Multi-Classes } \\
\hline [7] 2017 & SRs & $\begin{array}{c}\text { Functional Availability } \\
\text { Legal } \\
\text { Look and feel } \\
\text { Maintainability } \\
\text { Operational } \\
\text { Performance Scalability } \\
\text { Security } \\
\text { Usability } \\
\text { Fault tolerance } \\
\text { Portability }\end{array}$ & CNN & $\begin{array}{l}\text { PROMISE } \\
\text { corpus }\end{array}$ & $\begin{array}{l}\text { Precession: } 0.80 \\
\text { Recall: } 0.785 \\
\text { F-measure: } 0.77\end{array}$ & $\begin{array}{l}\text { SRs classified } \\
\text { into } 12 \text { classes } \\
\text { using DL. } \\
\text { SRs prepared } \\
\text { simply using } \\
\text { the proposed } \\
\text { reprocessing } \\
\text { method. } \\
\text { DL evaluated to } \\
\text { support RE. }\end{array}$ & $\begin{array}{l}\text { Limited to } \\
\text { CNN only, } \\
\text { while other } \\
\text { models could } \\
\text { be used. }\end{array}$ \\
\hline [18] 2018 & $\begin{array}{l}\text { 1. SRs } \\
\text { 2. NFRs } \\
\text { 3. NFRs }\end{array}$ & $\begin{array}{c}\text { 1. FRs } \\
\text { NFRs } \\
\text { 2. Availability } \\
\text { Legal } \\
\text { Maintainability } \\
\text { Operational } \\
\text { Performance } \\
\text { Scalability } \\
\text { Look and feel } \\
\text { Security usability } \\
\text { 3. Security } \\
\text { Not Security }\end{array}$ & $\mathrm{CNN}$ & $\begin{array}{l}\text { PROMISE } \\
\text { corpus }\end{array}$ & $\begin{array}{l}\text { 1. F1 score: } \\
0.945 \\
\text { 2. F1 score: } \\
0.911 \\
\text { 3. F1 score: } \\
0.772\end{array}$ & $\begin{array}{l}\text { Utilizing word } \\
\text { embedding in } \\
\text { vectorization } \\
\text { leads to } \\
\text { improvement in } \\
\text { SR } \\
\text { classification. }\end{array}$ & $\begin{array}{l}\text { Various } \\
\text { aspects } \\
\text { unexplored in } \\
\text { the method } \\
\text { and } \\
\text { validation. }\end{array}$ \\
\hline
\end{tabular}


Table 3. Cont.

\begin{tabular}{|c|c|c|c|c|c|c|c|}
\hline \multirow{2}{*}{$\begin{array}{c}\text { Reference \# } \\
\text { Year }\end{array}$} & \multicolumn{2}{|r|}{ Classification } & \multirow[t]{2}{*}{ Methodology } & \multirow[t]{2}{*}{ Dataset } & \multirow{2}{*}{ Results } & \multirow[t]{2}{*}{ Advantages } & \multirow[t]{2}{*}{ Disadvantages } \\
\hline & Input & Classes & & & & & \\
\hline [19] 2014 & SRs & $\begin{array}{c}\text { FR } \\
\text { Access control } \\
\text { Person authentication } \\
\text { Security encryption } \\
\text { Decryption Audit } \\
\text { control } \\
\text { Automatic Logoff } \\
\text { Integrity controls } \\
\text { Unique user } \\
\text { Identification } \\
\text { Transmission } \\
\text { Encryption Decryption } \\
\text { Emergency Access } \\
\text { procedure } \\
\text { Transmission Security }\end{array}$ & $\begin{array}{c}\text { WekaClassifiersTrees } \\
\text { REPTree } \\
\text { J48 }\end{array}$ & $\begin{array}{c}\text { Certification } \\
\text { Commission for } \\
\text { Healthcare } \\
\text { Information } \\
\text { Technology (CCHIT) } \\
\text { dataset }\end{array}$ & $\begin{array}{l}\text { Accuracy: } \\
93.92 \%\end{array}$ & $\begin{array}{l}\text { ML algorithms } \\
\text { combined to } \\
\text { solve } 2 \mathrm{RE} \\
\text { problems, } \\
\text { and more } \\
\text { problems can } \\
\text { be solved. }\end{array}$ & $\begin{array}{l}\text { Additional } \\
\text { components } \\
\text { required to } \\
\text { solve RE } \\
\text { problems with } \\
\text { Tracelab. }\end{array}$ \\
\hline [12] 2013 & NFRs & $\begin{array}{c}14 \text { NFR classes such as: } \\
\text { Capacity } \\
\text { Reliability } \\
\text { Security }\end{array}$ & $\begin{array}{l}\text { K-nearest neighbor } \\
\text { classifier }\end{array}$ & $\begin{array}{l}11 \text { documents related } \\
\text { to electronic health } \\
\text { records (EHRs) }\end{array}$ & F1: 0.54 & $\begin{array}{l}\text { Ability to } \\
\text { extract relevant } \\
\text { NFRs from } \\
\text { documents. }\end{array}$ & $\begin{array}{l}\text { Limited to } \\
\text { NFRs. } \\
\text { Specific data } \\
\text { field. }\end{array}$ \\
\hline [13] 2007 & SR & $\begin{array}{c}\text { FRs } \\
\text { Availability } \\
\text { Look and-feel } \\
\text { legal } \\
\text { Maintainability } \\
\text { Operational } \\
\text { Performance } \\
\text { Scalability } \\
\text { Security } \\
\text { Usability }\end{array}$ & $\begin{array}{l}\text { Classification based } \\
\text { on key words for each } \\
\text { category, along with } \\
\text { finding the weight } \\
\text { based on frequency } \\
\text { for each keyword } \\
\text { used by TF-IDF }\end{array}$ & $\begin{array}{l}\text { Labeled } 326 \text { NFRs } \\
\text { and } 358 \text { FRs from } 15 \\
\text { different projects }\end{array}$ & $\begin{array}{l}\text { Considering } \\
\text { only the top } 15 \\
\text { keywords for } \\
\text { each category } \\
\text { scored the } \\
\text { highest } \\
\text { Recall: } 0.7669\end{array}$ & $\begin{array}{l}\text { Provided an } \\
\text { incremental } \\
\text { approach for } \\
\text { training a } \\
\text { classifier in new } \\
\text { domains. }\end{array}$ & $\begin{array}{l}\text { More efforts to } \\
\text { improve } \\
\text { stopping } \\
\text { conditions. } \\
\text { Run time } \\
\text { needs to be } \\
\text { improved to } \\
\text { be used by a } \\
\text { real analyst. }\end{array}$ \\
\hline [14] 2010 & SR & $\begin{array}{c}\text { FRs } \\
\text { Availability } \\
\text { Look and feel } \\
\text { Legal } \\
\text { Maintainability } \\
\text { Operational } \\
\text { Performance Scalability } \\
\text { Security } \\
\text { Usability }\end{array}$ & Naïve Bayes classifier & $\begin{array}{l}\text { Labeled } 326 \text { NFRs } \\
\text { and } 358 \text { FRs from } 15 \\
\text { different projects }\end{array}$ & Accuracy: 97\% & $\begin{array}{l}\text { Feasibility of } \\
\text { using semi- } \\
\text { supervised } \\
\text { learning in NFR } \\
\text { classification. }\end{array}$ & $\begin{array}{l}\text { Dataset is } \\
\text { limited to the } \\
\text { top-ranked } \\
\text { examples. }\end{array}$ \\
\hline [15] 2019 & SR & $\begin{array}{c}\text { FRs } \\
\text { NFRs categorized into } \\
10 \text { categories such as: } \\
\text { Security } \\
\text { Performance } \\
\text { Usability }\end{array}$ & $\begin{array}{l}\text { Ensemble method } \\
\text { consists of random } \\
\text { forest and gradient } \\
\text { boosting }\end{array}$ & $\begin{array}{l}\text { Text files in a format } \\
\text { of SQL and CSV files }\end{array}$ & $\begin{array}{l}\text { NFR accuracy: } \\
0.826 \text { (gradient } \\
\text { boosting) } \\
\text { FR accuracy: } \\
0.591 \text { (random } \\
\text { forest) }\end{array}$ & $\begin{array}{l}\text { Highly accurate } \\
\text { algorithms for } \\
\text { SR } \\
\text { classification. }\end{array}$ & $\begin{array}{c}\text { Limited study } \\
\text { due to limited } \\
\text { data. } \\
\text { Could not } \\
\text { determine the } \\
\text { number of SRs } \\
\text { in a sentence. } \\
\text { Could not } \\
\text { read input } \\
\text { from .docx } \\
\text { and .txt files. }\end{array}$ \\
\hline [16] 2010 & SR & $\begin{array}{c}\text { FRs } \\
\text { NFRs categorized into } \\
\text { categories such as: } \\
\text { Security } \\
\text { Availability } \\
\text { Scalability }\end{array}$ & Naïve Bayes classifier & $\begin{array}{c}\text { Collected SRs by } \\
\text { interviews and other } \\
\text { methods } \\
\text { (255 FRs, NFRFR } \\
\text { range from } 10 \text { to } 67 \\
\text { per category) }\end{array}$ & $\begin{array}{l}\text { NFR precision: } \\
75 \%\end{array}$ & $\begin{array}{c}\text { Reduced } \\
\text { manual labeling } \\
\text { efforts. }\end{array}$ & $\begin{array}{l}\text { Limited to } \\
\text { NFR } \\
\text { multi-class } \\
\text { classification }\end{array}$ \\
\hline [17] 2019 & SR & $\begin{array}{c}\text { FRs } \\
\text { Availability } \\
\text { Legal } \\
\text { Look and feel } \\
\text { Maintainability } \\
\text { Operational } \\
\text { Performance } \\
\text { Scalability } \\
\text { Security } \\
\text { Usability } \\
\text { Fault tolerance } \\
\text { Portability }\end{array}$ & $\begin{array}{c}\text { Multinomial naïve } \\
\text { Bayes (MNB) } \\
\text { Gaussian naïve Bayes } \\
\text { (GNB) } \\
\text { Bernoulli naïve Bayes } \\
\text { (BNB) } \\
\text { K-nearest neighbor } \\
\text { (KNN) } \\
\text { Support vector } \\
\text { machine (SVM) } \\
\text { Stochastic gradient } \\
\text { descent SVM (SGD } \\
\text { SVM) } \\
\text { Decision tree (Dtree) }\end{array}$ & $\begin{array}{l}\text { PROMISE dataset } \\
\text { (625 requirement } \\
\text { sentences: } 255 \text { FRs } \\
\text { and } 370 \text { NFRs) }\end{array}$ & $\begin{array}{l}\text { Precision: } 0.66 \\
\text { Recall: } 0.61 \\
\text { F1 score: } 0.61 \\
\text { Accuracy: } 0.76 \\
\text { Using SGD } \\
\text { SVM classifier } \\
\text { and TF-IDF for } \\
\text { feature } \\
\text { extraction }\end{array}$ & $\begin{array}{l}\text { Seven } \\
\text { algorithms } \\
\text { used. }\end{array}$ & $\begin{array}{l}\text { Need to apply } \\
\text { other } \\
\text { classification } \\
\text { techniques } \\
\text { such as } \\
\text { boosting and } \\
\text { bagging. }\end{array}$ \\
\hline
\end{tabular}


Table 3. Cont.

\begin{tabular}{|c|c|c|c|c|c|c|c|}
\hline \multirow{2}{*}{$\begin{array}{c}\text { Reference \# } \\
\text { Year }\end{array}$} & \multicolumn{2}{|c|}{ Classification } & \multirow{2}{*}{ Methodology } & \multirow{2}{*}{ Dataset } & \multirow{2}{*}{ Results } & \multirow{2}{*}{ Advantages } & \multirow{2}{*}{ Disadvantages } \\
\hline & Input & Classes & & & & & \\
\hline \multicolumn{8}{|c|}{ 4. Complete System (Classifying NFRs and FRs into Multi-Classes) } \\
\hline [20] 2020 & $\begin{array}{l}\text { 1. SRs } \\
\text { 2. NFRs } \\
\text { 3. FRs }\end{array}$ & $\begin{array}{l}\text { 1. FRs } \\
\text { NFRs } \\
\text { 2. Usability } \\
\text { Security } \\
\text { Operational } \\
\text { Performance } \\
\text { 3. Functions } \\
\text { Data Behavior }\end{array}$ & $\begin{array}{l}\text { Fine-tuned BERT } \\
\text { (NoRBERT) }\end{array}$ & PROMISE & $\begin{array}{l}\text { 1. F1 score: } 90 \% \\
\text { FRs and } 93 \% \\
\text { NFRs } \\
\text { 2. F1 score: } 76 \% \\
\text { 3. F1 score: } 92 \%\end{array}$ & $\begin{array}{l}\text { Novelty in FR } \\
\text { classification. } \\
\text { Modern } \\
\text { algorithm used } \\
\text { with transfer } \\
\text { learning. }\end{array}$ & $\begin{array}{l}\text { Multi-class or } \\
\text { multi-label } \\
\text { classification } \\
\text { for FRs not } \\
\text { considered. }\end{array}$ \\
\hline
\end{tabular}

\section{Materials and Methods}

\subsection{Phases of Classification}

In this section, the phases of classifications are explained, as shown in Figure 1. First, SRs are classified into FRs or NFRs, and this is called binary classification. Second, FRs are classified into six different classes, as explained in detail in Table 1. The multi-classes of FRs include: solution, enablement, action constraint, attribute constraint, policy, and Definition. Third, NFRs are classified into 11 different classes, as summarized in Table 2. The multiclasses of NFRs include availability, fault tolerance, Legal, look and feel, maintainability, operational, performance, portability, scalability, usability, and security. In order to conduct these classifications, two experiments were conducted and are explained in the following sections.

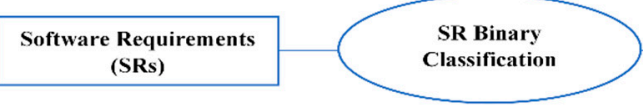

(SRs)
(SRu

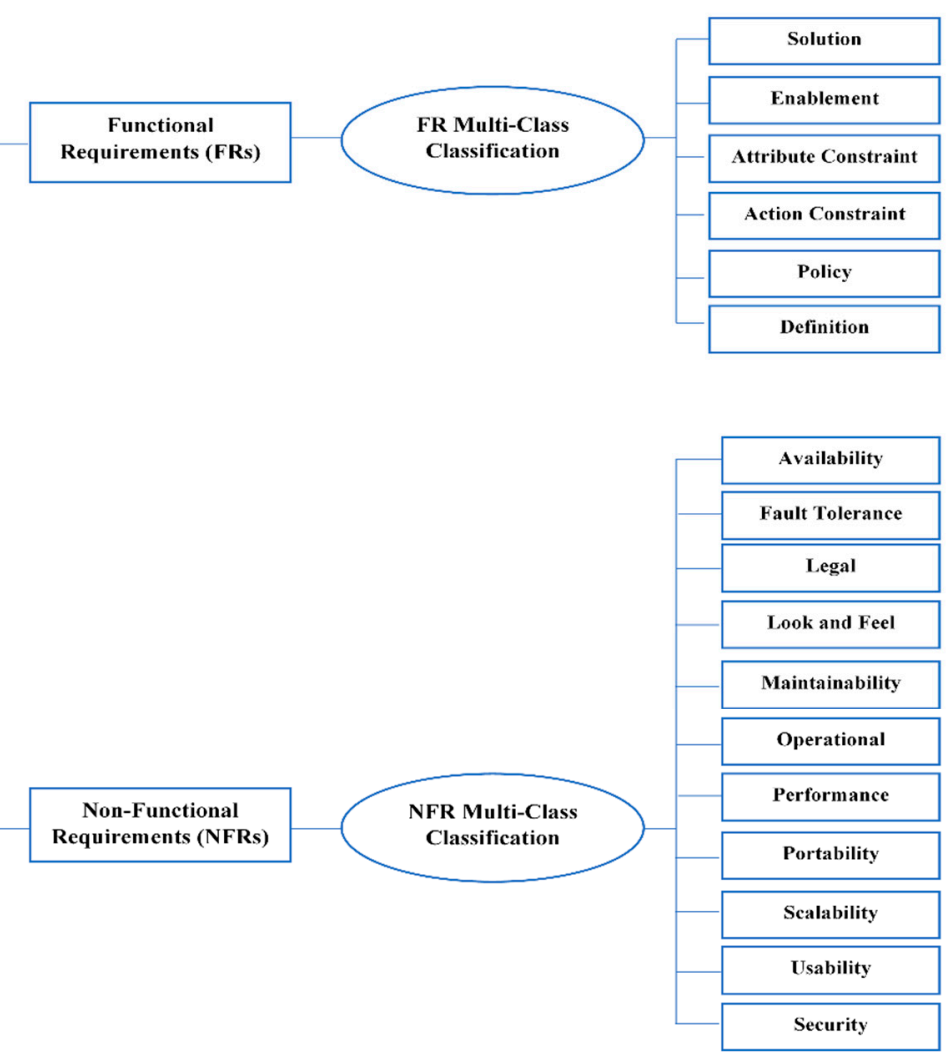

Figure 1. Phases of software requirement (SR) classification.

\subsection{Methodology}

In this section, the proposed models are explained in detail. Figure 2 depicts the two models, which include the following phases, and the details are included in the subsequent sections: 
- Data preprocessing;

- Text classification phase 1 (binary);

- Text classification phase 2 (multi-classes);

- Evaluation.

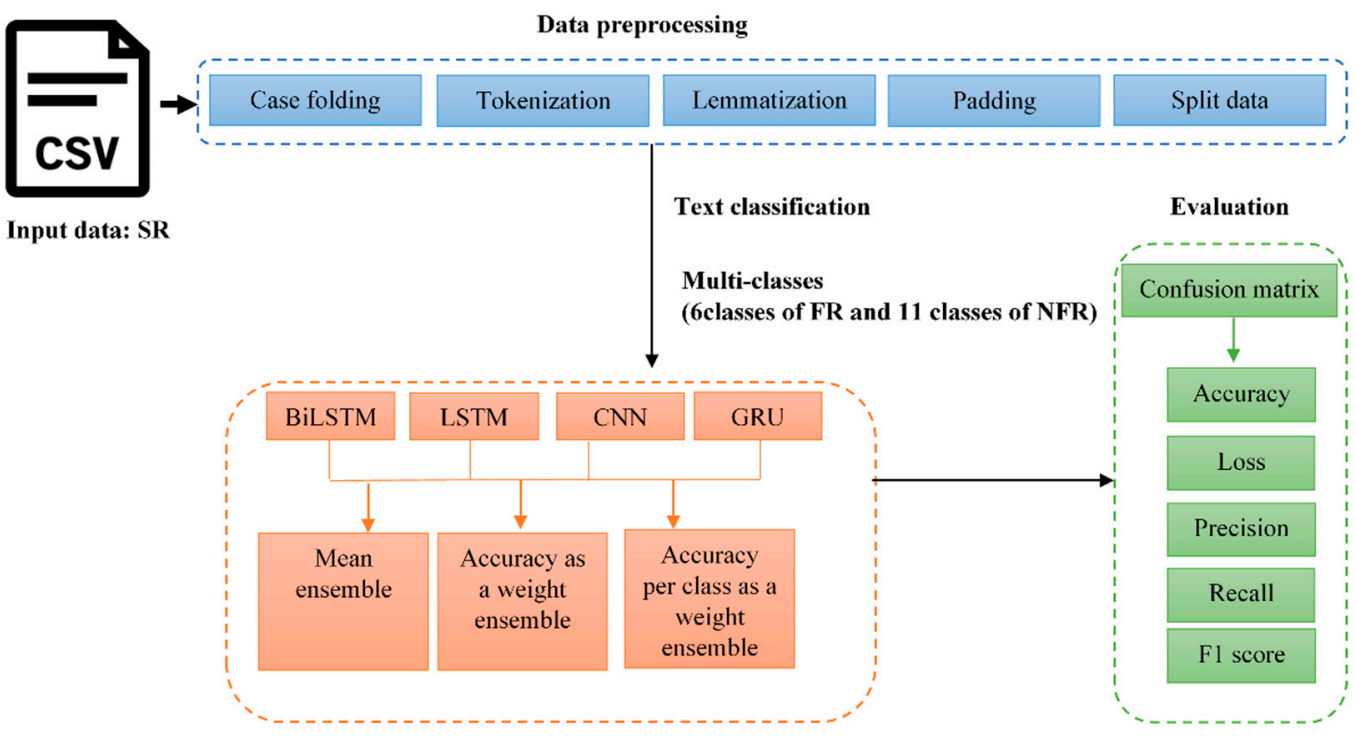

(a)

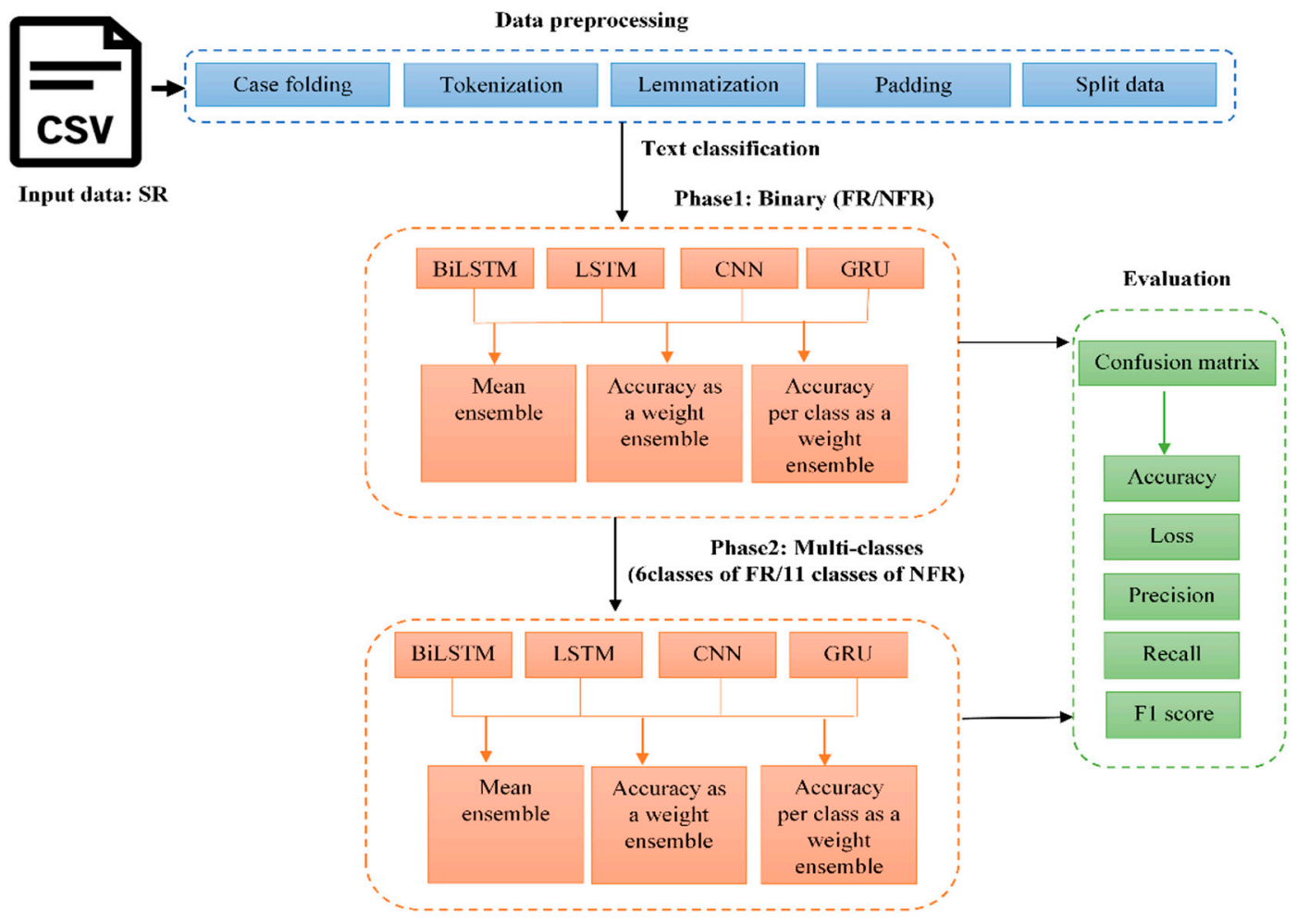

(b)

Figure 2. Proposed models (a) One phase (b) Two phases. 


\subsubsection{Data Preprocessing}

This process is conducted only once at the beginning before phase 1 classification. Data preprocessing is a crucial step in DL models, as it affects the accuracy and quality of results. The preprocessing for the DL models was performed in a unified way. The preprocessing included four steps: (a) Case folding, (b) tokenization, (c) lemmatization, and (d) padding. The text was converted to lowercase in order to avoid having two dimensions for the same words [7]. Tokenization refers to converting the text to tokens of words [19]. Lemmatization means returning each word to its root and finding the correct base word for it, and it was selected because it outperforms stemming [21]. Finally, padding was used to unify the length of the sentences, since they had different numbers of words. This was carried out by finding the maximum length of the sentence and then adding zeros to the end of the sequence of tokens for any sentence that was shorter than the maximum length specified according to the input [20]. Algorithm 1 summarizes the preprocessing and Figure 3 gives an example of preprocessing of one SR from the dataset.
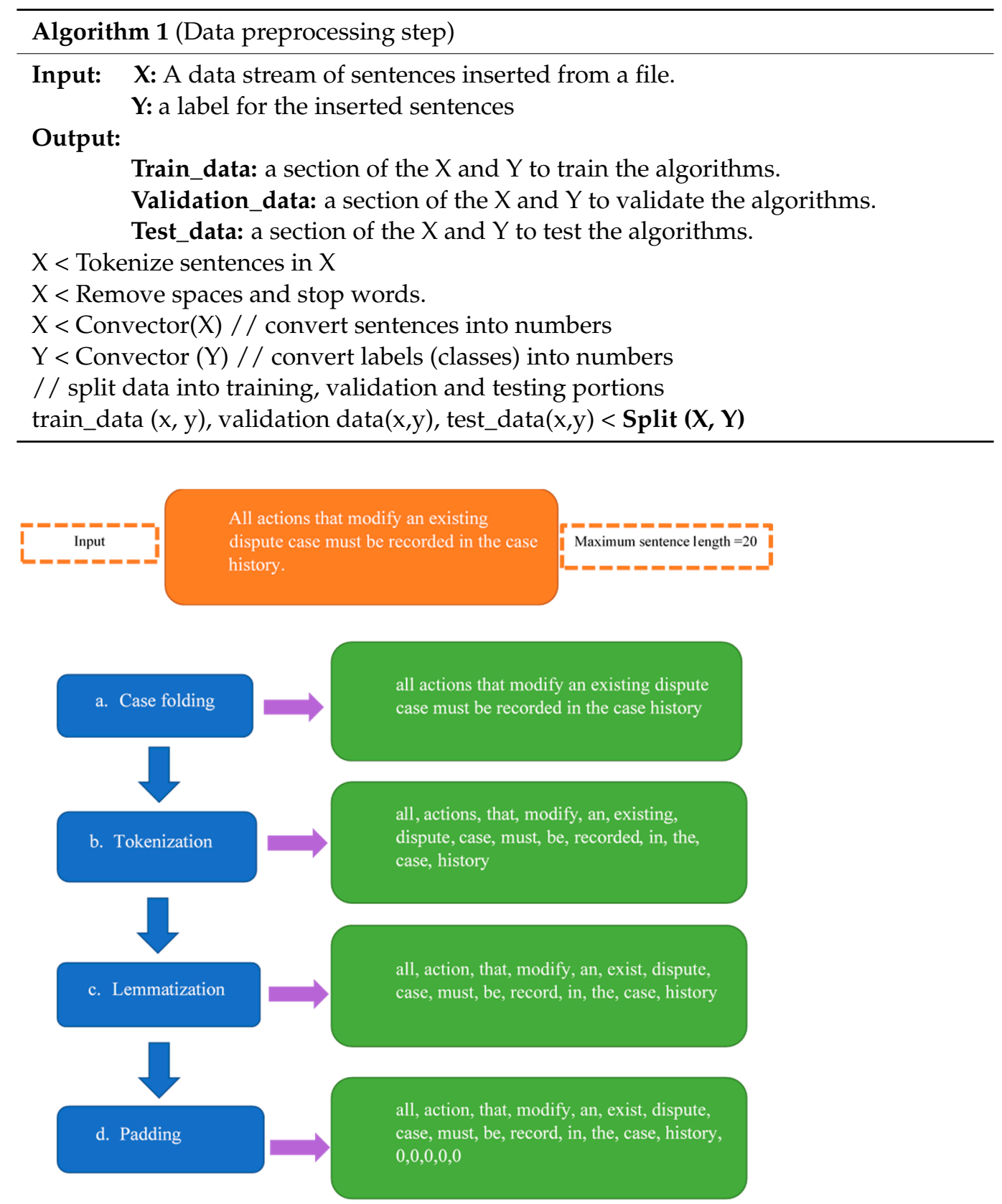

Figure 3. Preprocessing example. 


\subsubsection{Text Classification}

A. Base DL Classifiers:

The following are the used base DL classifiers in both the binary and multi-class classifications:

- $\quad$ LSTM is a type of recurrent neural network (RNN) that uses memory blocks to solve the vanishing gradient problem in RNN using memory blocks. The model's first layer is the input layer, which receives preprocessed data in a time step. Each component is passed to the embedding layer at first, which is represented to generate feature vectors. Then, the LSTM hidden layer follows the forward path only. LSTM has three main gates, namely, input, forget, and output gates, to control the cell state and update the weights [22].

- $\quad$ BiLSTM includes two hidden layers connected with both the input and the output. BiLSTM includes a forward LSTM layer and a backward LSTM layer to utilize the next tokens for learning information, and better predictions can be achieved. The best way to benefit from the BiLSTM is to stack LSTM layers. Forward layers are iterated from $t=1$ to $T$. On the contrary, backward layers are iterated from $t=T$ to 1 [23].

- $\quad$ CNNs involve producing local features by applying the convolutional concept [24]. Using filters with a width determined by the word embedding vector size, different vertical local regions allow different filter sizes of $L=2,3$, and 4 . This is helpful for learning many features. Active convoluted results are used to generate feature maps with varied dimensions on the filters [25].

- GRU is type of RNN known for its fast conversions compared to LSTM. In addition, it requires fewer parameters [26]. It has two types of gates: An update gate and a reset gate. Since it has no memory to store information, it only deals with unit information. The update gate decides the amount of data to be updated and the reset gate decides the amount of past data to forget. If the gate is set to zero, it reads input data and ignores the previously calculated state [27].

B. Ensemble Models:

A number of ensemble methods are applied to the specified dataset (PROMISE). Then, the performance is compared. Details of each ensemble model are explained in the subsections of section B (B.1, B.2, and B.3).

\section{B.1. Mean Ensemble:}

This ensemble is also called simple averaging. The output is the average prediction from base classifiers that have equal weights [28]. The mean ensemble is explained in Algorithm 2.

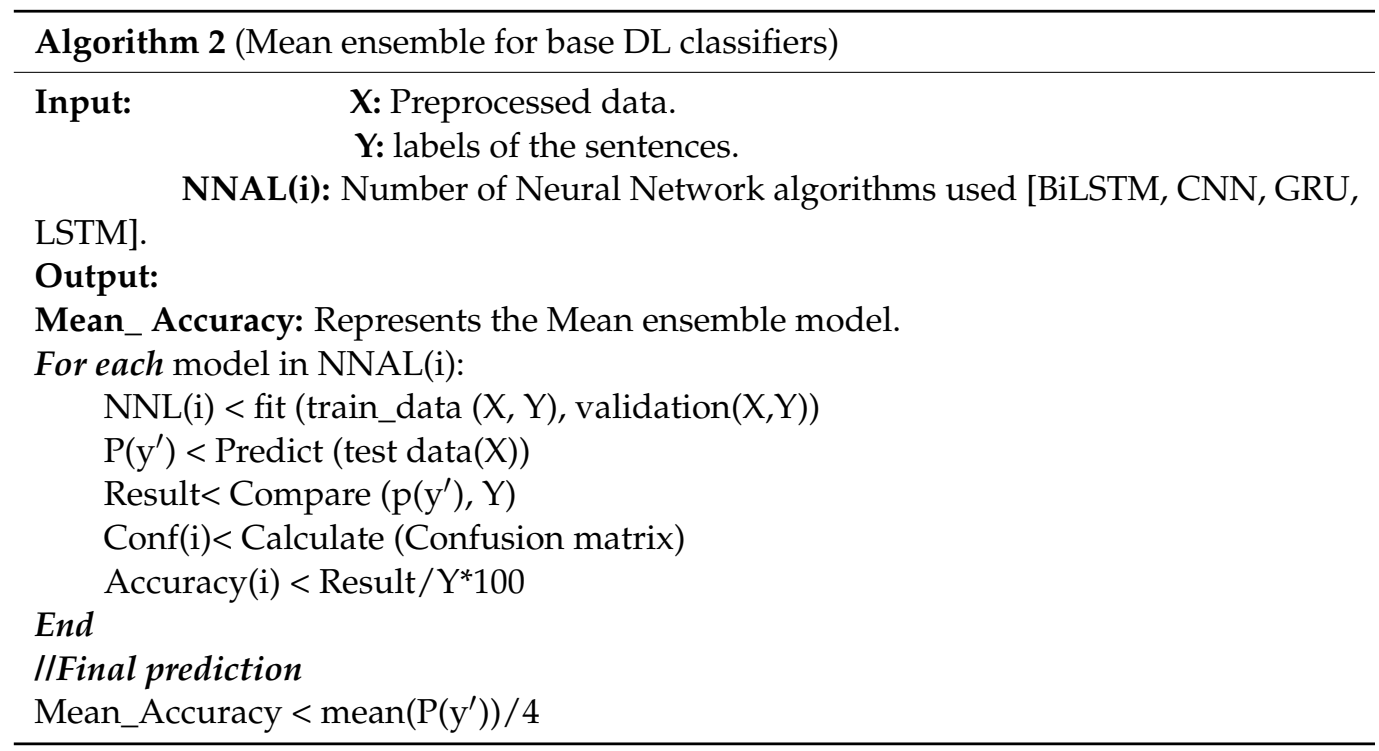




\section{B.2. Accuracy as a weight:}

This method is a combination of weight ensemble and simple averaging ensemble. The prediction of each model is multiplied by its weight, which is the accuracy in this case. Then, the average is calculated [29]. Algorithm 3 displays the accuracy as a weight ensemble.

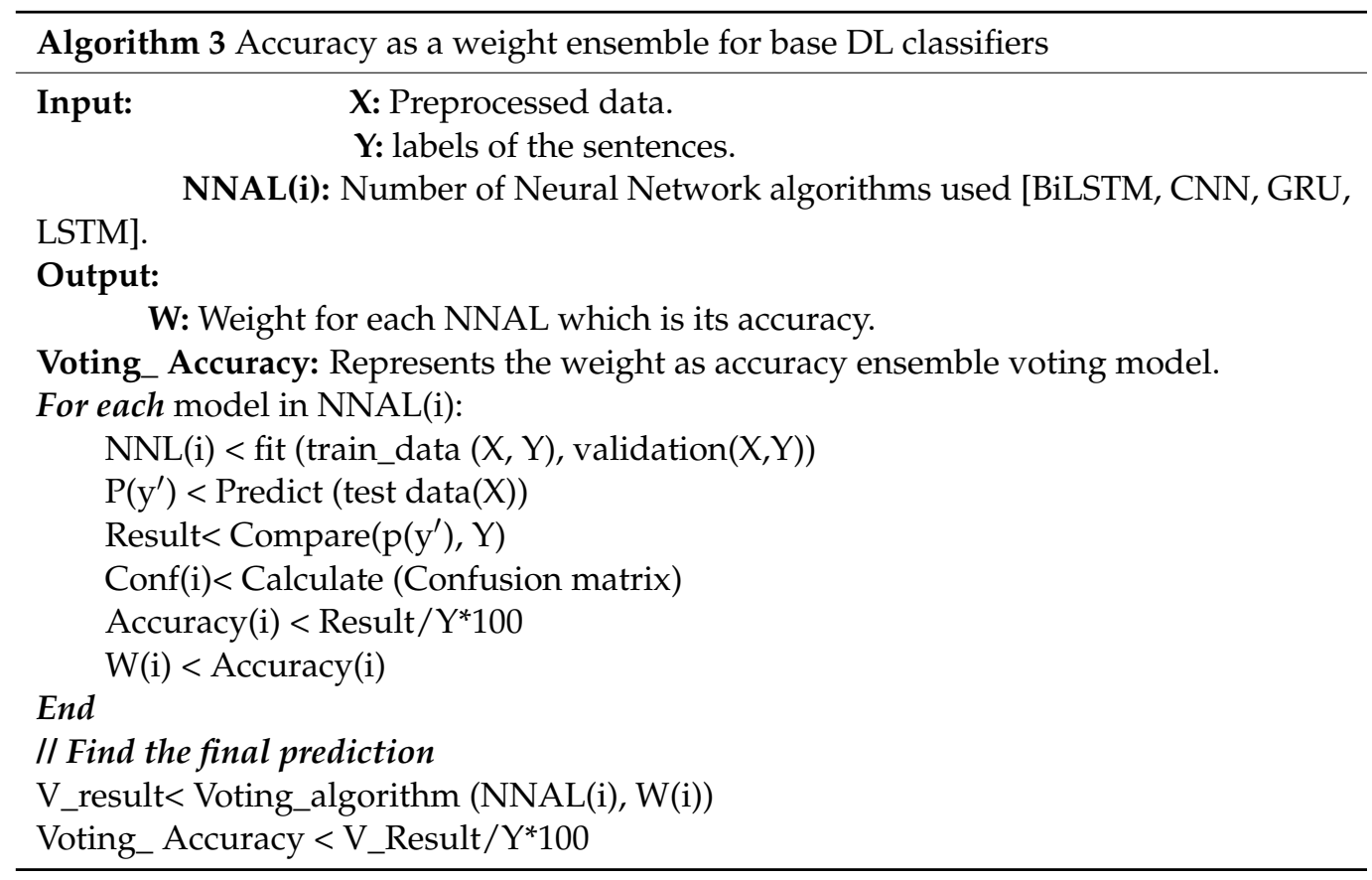

B.3. Accuracy per class as a weight:

This ensemble classifier is an enhancement of the voting ensemble, considering the accuracy as a weight. The enhancement consists of calculating the accuracy per class and passing it to the voting ensemble, rather than the overall accuracy, as other authors have done. This method of ensemble was proposed by [11], who applied the concept to ML classifiers. Figure 3 explains this ensemble method as having two base classifiers: Classifiers A and B. The type of classification is multi-class classification into class 0,1 , or 2 . As per the example in Figure 4, the confusion matrices are created for base classifiers. Then, predictions for each class by each base classifier are stored as a matrix to be compared. For this example, for class 0, Classifier A prediction is better than Classifier B, so it is considered. For class 1, both classifiers have the same predictions, so one of them is selected randomly. Classifier B scores better prediction for class 2, resulting in it being considered. The better predictions for each class are considered to form the final confusion matrix. Algorithm 4 explains the accuracy per class as a weight ensemble model.

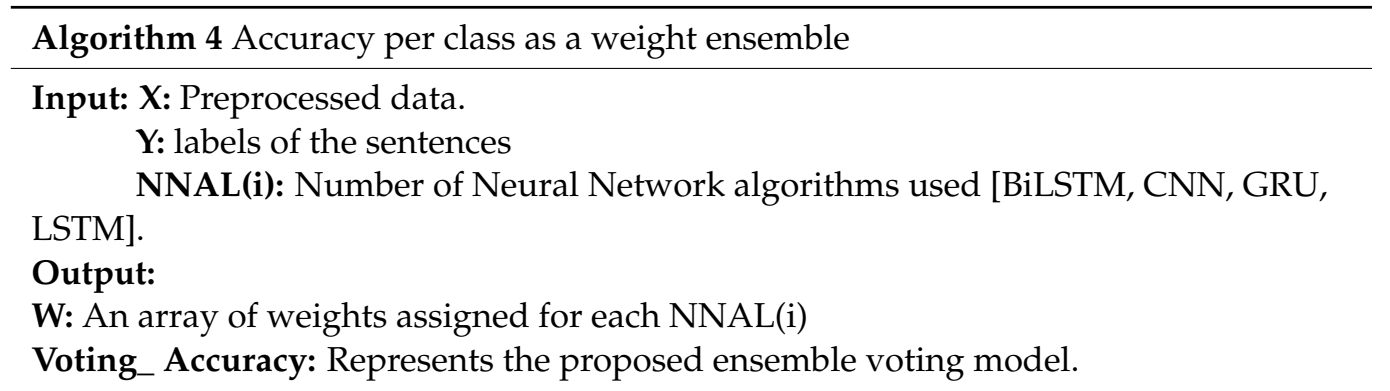


For each model in NNAL(i):

$\mathrm{NNL}(\mathrm{i})<$ fit (train_data $(\mathrm{X}, \mathrm{Y})$, validation_data $(\mathrm{X}, \mathrm{Y}))$

$\mathrm{P}\left(\mathrm{y}^{\prime}\right) \quad<$ Predict (test $\left.\operatorname{data}(\mathrm{X})\right)$

Result $<$ Compare $\left(\mathrm{p}\left(\mathrm{y}^{\prime}\right), \mathrm{Y}\right)$

Conf(i) $<$ Calculate (Confusion matrix)

Accuracy(i) $<$ Result $/ Y^{*} 100$

End

// Give a weight for each model

// combine the diagonals of all confusion matrices into one matrix

Conf_matrix $<$ [[Conf (i. diagonal]]

/ find the maximum of each column that will represent the algorithm weight

$\mathrm{W}<$ max_coloumn (Conf_matrix(i))

V_result $<$ Voting_algorithm (NNAL(i), W(i))

Voting_Accuracy $<$ V_Result $/ Y^{*} 100$

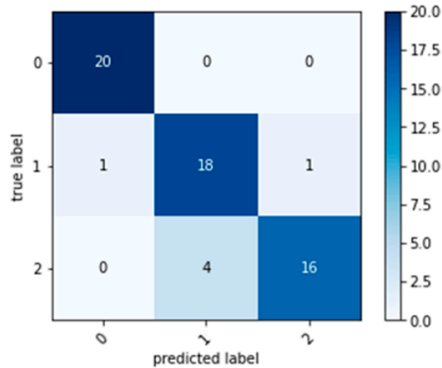

Classifier A

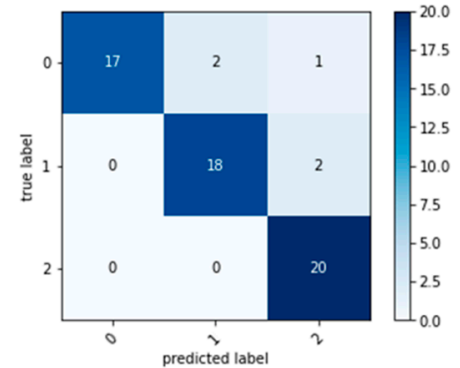

Classifier B

(a)

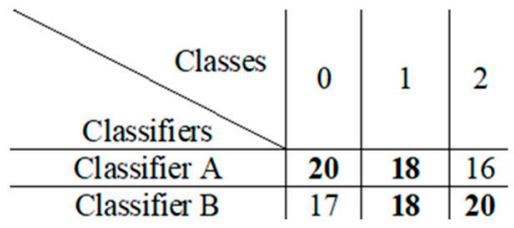

(b)

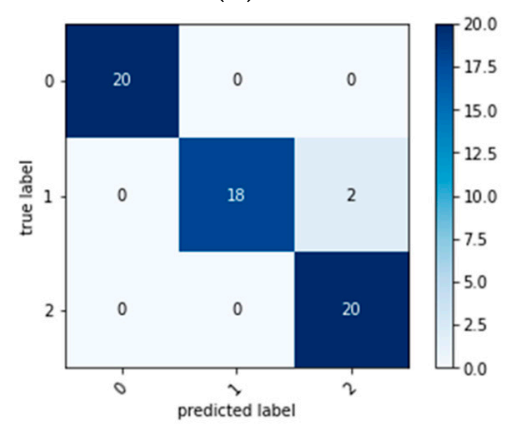

(c)

Figure 4. Example of accuracy per class as a weight. (a) confusion matrices for base classifiers; (b) matrix to store predictions of each class by each classifier (diagonals of confusion matrices); (c) confusion matrix of the final ensemble.

\subsubsection{Evaluation Methods}

Evaluation was conducted using different performance measures:

\section{A. Confusion Matrix}

The evaluation of each DL classifier was based on the confusion matrix, which summarized the correct and incorrect predictions of each classifier for each class, showing a ratio of 
correct predictions to the total predictions made by the classifier. The parameters that were required to calculate the other evaluation matrices were calculated through the confusion matrix. These parameters were the true positive (tp), false positive (fp), true negative (tn), and false negative (fn). The predicted classes are represented in rows, while actual classes are represented in columns [30].

\section{B. Accuracy}

Accuracy is one of the most important evaluation matrices, showing the overall performance of a classifier. The following formula was used to calculate the accuracy [31]:

$$
\text { Accuracy }=\frac{\mathrm{tp}+\mathrm{tn}}{\mathrm{tp}+\mathrm{tn}+\mathrm{fp}+\mathrm{fn}}
$$

where tp refers to true positive predictions, $t \mathrm{n}$ refers to true negative predictions, fp refers to false positive predictions, and fn refers to false negative predictions [31].

C. Loss

Loss was used to calculate the difference between predictions and true values. In the case that each input belonged to one category from the multiple categories, "sparse_categorical_ crossentropy" was used. The following formula clarifies this calculation, where S refers samples, $C$ refers to classes, and $(S \in C)$ represents the samples belonging to class $C$ [32]:

$$
\text { Loss }=-\log \mathrm{p}(\mathrm{S} \in \mathrm{C})
$$

D. Precision

The precision is the total of the positive prediction values and was calculated using the following formula [32]:

$$
\text { Precision }=\frac{\mathrm{tp}}{\mathrm{tp}+\mathrm{fp}}
$$

where tp refers to true positive predictions and fp refers to false positive predictions.

E. Recall

Recall is defined as sensitivity or the true positive rate and was calculated using the following formula [32]:

$$
\text { Recall }=\frac{\mathrm{tp}}{\mathrm{tp}+\mathrm{fn}}
$$

where tp refers to true positive predictions and fn refers to false negative predictions.

F. F1 Score

The F1 score is a relationship metric between recall and precision. It can be high only if both recall and precision are high. The F1 score was calculated using the following formula [32]:

$$
\mathrm{F} 1-\text { Score }=2 * \frac{\text { Precision } * \text { Recall }}{\text { Precision }+ \text { Recall }}
$$

\section{Experiment Details}

\subsection{Hardware and Software Details}

Python 3.6 was used for implementing the base and ensemble classifier. We used the PyCharm tool, which is user-friendly, and its dataset allows for easy uploading. This is true for various dataset file formats, such as CSV.

Scikit learn was also chosen, as it includes many libraries that can facilitate building ML classifiers and calculating the factors used in the evaluations. Ensemble models are widely supported by all types of classifiers.

The computer used was an ASUS Laptop, with an x64 Inter(R) Core (TM) i7-9750H processor and 17.0 GB of RAM, running a 64-bit Windows Operating System. 


\subsection{PROMISE Dataset}

This is a widely used dataset for SR classification in most previous work. It consists of FRs and NFRs labeled into 11 different categories, as explained in Table 1, with a different number of statements in each class. The content of the found and used version of the PROMISE dataset is detailed in Table 4.

Table 4. PROMISE dataset details.

\begin{tabular}{cc}
\hline Classes & Number of Statements \\
\hline Availability (A) & 29 \\
Fault tolerance (FT) & 12 \\
Legal (L) & 14 \\
Look and feel (LF) & 42 \\
Maintainability (MN) & 17 \\
Operational (O) & 68 \\
Performance (PE) & 54 \\
Portability (PO) & 2 \\
Scalability (SC) & 21 \\
Security (SE) & 67 \\
Usability (US) & 67 \\
FRs & 266 \\
Total & 659 \\
\hline
\end{tabular}

The FRs were labeled into six classes by the authors to be used in the experiments, containing different numbers in each class, as shown in Table 5.

Table 5. Details of the PROMISE FR classes.

\begin{tabular}{cc}
\hline FR Classes & Number of Statements \\
\hline Policy (P) & 41 \\
Solution (S) & 116 \\
Enablement (E) & 71 \\
Action constraint (AC) & 25 \\
Attribute constraint (AT) & 7 \\
Definition (D) & 6 \\
Total & 266 \\
\hline
\end{tabular}

The split of the dataset into training, validation, and testing was unified for all systems and experiments to $35 \%$ training, 35\% validation, and 30\% testing. An imbalanced dataset has different solutions to form a balanced dataset for experiments. The selected solution according to the available dataset was random oversampling. This technique duplicates minority classes in training datasets and is repeated until satisfaction of class distribution is reached [32].

\subsection{One-Phase Classification System}

A. Training

This system classifies SRs into one of the FR or NFR classes (17 classes) directly without passing the phase of binary classification at the beginning. The validation dataset was used to ensure that there is no overfitting in each base DL classifier. The hyperparameters were left to the default and some were justified to the values that help to avoid overfitting. The Adam optimizer was used with a 0.001 default learning rate. The suitable number of epochs was 30 with batch size of 50. Dropout was justified to 0.2 for all classifiers. The architecture of each base DL model is summarized in Tables 6-9.

Figure 5 shows the accuracy and loss for DL models during 60 epochs to find the optimal number of epochs. As it was noticed that 30 epochs is optimal for all DL models, this number of epochs was selected for all experiments. 


\section{B. Test Results and Discussion}

Table 10 summarizes the evaluation parameters in the testing phase for SR multi-class classification. The base DL models reached a $91 \%$ accuracy, a 0.92 precision, a 0.92 recall, and a $0.92 \mathrm{~F} 1$ score by the $\mathrm{CNN}$ model. The applied ensemble methods reached a $92.56 \%$ accuracy, a 0.93 precision, a 0.93 recall, a 0.93 F1 score by accuracy per class as a weight ensemble method. The confusion matrices of all models and methods applied on the PROMISE dataset are displayed in Figure 6, where it can be noticed that the most accurate predictions for all classes were reached by the accuracy per class as a weight ensemble method. The ensemble method was able to enhance the accuracy of the SR classification, but the authors would prefer to examine the application phases of classification on the PROMISE dataset to find the best approach—either a one- or two-phase system.

Table 6. One-phase system: architecture of the BiLSTM DL model.

\begin{tabular}{ccc}
\hline Layer & Output Shape & Number of Parameters \\
\hline Embedding_64 & (None, None, 46) & 77,280 \\
Spatial_dropout1d_39 & (None, None, 46) & 0 \\
Bidirectional_39 & (None, 92) & 34,224 \\
Dense_149 & (None, 46) & 4278 \\
Dropout_95 & (None, 46) & 0 \\
Dense_150 & (None, 46) & 2162 \\
Dropout_96 & (None, 46) & 0 \\
Dense_151 & (None, 17) & 799 \\
Activation_39 & (None, 17) & 0 \\
\hline
\end{tabular}

Table 7. One-phase system: Architecture of the CNN model.

\begin{tabular}{ccc}
\hline Layer & Output Shape & Number of Parameters \\
\hline Embedding_65 & (None, None, 46) & 77,280 \\
Dropout_97 & (None, None, 46) & 0 \\
Conv1d_10 & (None, None, 46) & 6394 \\
Global_max_pooling1d_10 & (None, 46) & 0 \\
Dense_152 & (None, 128) & 6016 \\
Dropout_98 & (None, 128) & 0 \\
Dense_153 & (None, 17) & 2193 \\
\hline
\end{tabular}

Table 8. One-phase system: architecture of the GRU model.

\begin{tabular}{ccc}
\hline Layer & Output Shape & Number of Parameters \\
\hline Embedding_66 & (None, None, 46) & 77,280 \\
Gru_17 & (None, None, 46) & 12,834 \\
Gru_18 & (None, 32) & 7584 \\
Dense_154 & (None, 17) & 561 \\
\hline
\end{tabular}

Table 9. One-phase system: Architecture of the LSTM model.

\begin{tabular}{ccc}
\hline Layer & Output Shape & Number of Parameters \\
\hline Embedding_67 & (None, None, 46) & 77,280 \\
Lstm_56 & (None, None, 46) & 17,112 \\
Lstm_57 & (None, 32) & 10,112 \\
Dense_155 & (None, 17) & 561 \\
\hline
\end{tabular}


Table 10. One-phase system: evaluation parameters for the DL models.

\begin{tabular}{ccccc}
\hline DL Models & $\begin{array}{c}\text { Accuracy } \\
\mathbf{( \% )}\end{array}$ & Precision & Recall & F1 Score \\
\hline BiLSTM & 84.022 & 0.84 & 0.85 & 0.84 \\
LSTM & 74.656 & 0.75 & 0.74 & 0.72 \\
CNN & 91 & 0.92 & 0.92 & 0.92 \\
GRU & 78.78 & 0.79 & 0.79 & 0.78 \\
Mean ensemble & 88.15 & 0.88 & 0.89 & 0.88 \\
Accuracy as a weight ensemble & 90.63 & 0.91 & 0.91 & 0.91 \\
Accuracy per class as a weight & 92.56 & 0.93 & 0.93 & 0.93 \\
\hline
\end{tabular}
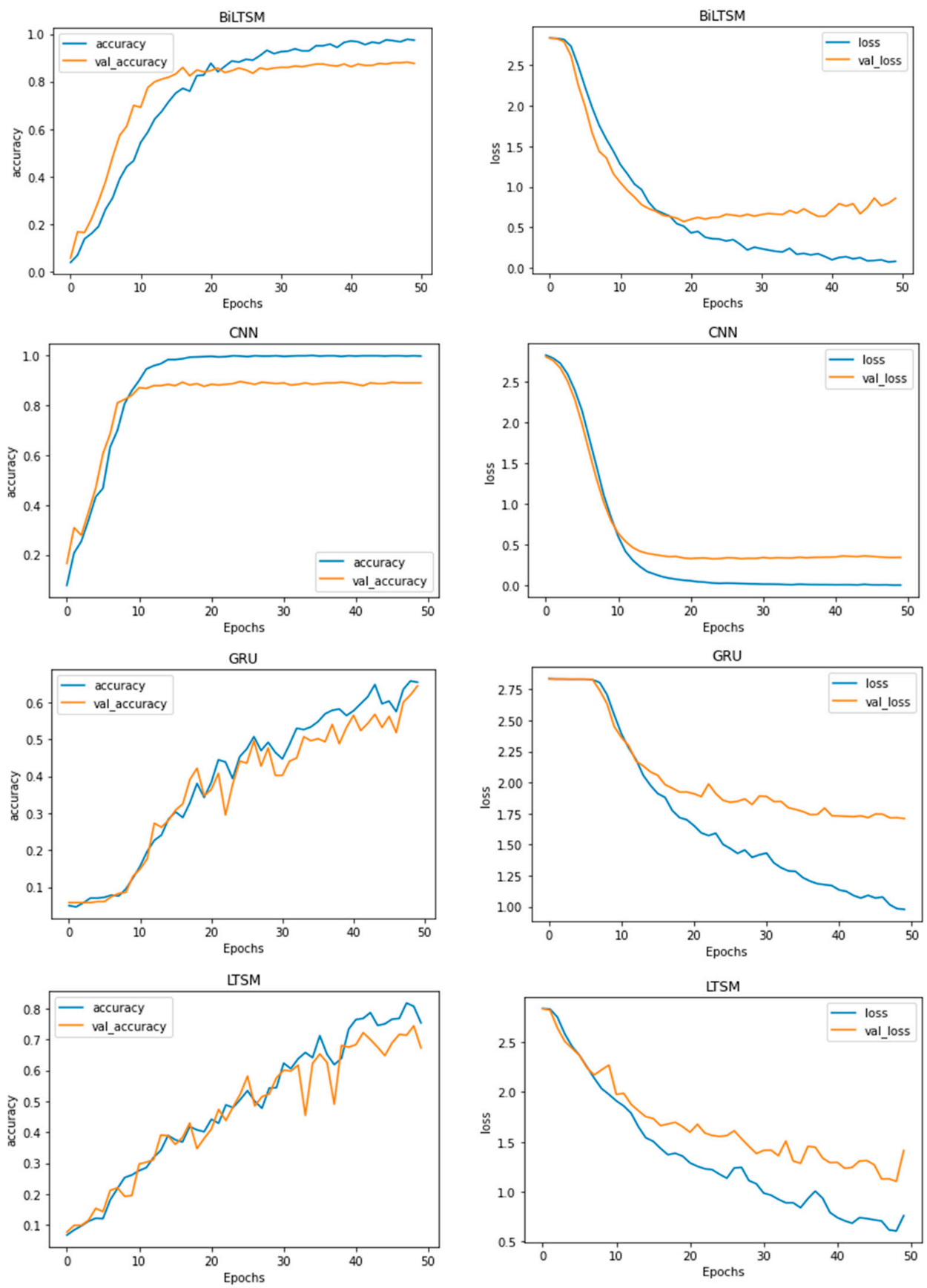

Figure 5. Accuracy-loss curves for the DL models in 60 epochs. 


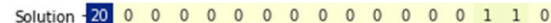
Enablement - $0 \begin{array}{llllllllllllllllllll}11 & 0 & 0 & 0 & 0 & 0 & 0 & 0 & 1 & 0 & 0 & 0 & 0 & 0 & 0 & 0\end{array}$ Policy - $0 \begin{array}{lllllllllllllllllll}0 & 19 & 0 & 1 & 0 & 0 & 0 & 1 & 0 & 0 & 1 & 0 & 0 & 0 & 0 & 0\end{array}$ Action - $0 \begin{array}{llllllllllllllll}10 & 0 & 22 & 0 & 0 & 0 & 0 & 0 & 0 & 0 & 0 & 0 & 0 & 0 & 0 & 0\end{array}$ Action - $0 \begin{array}{lllllllllllllllll}0 & 0 & 0 & 12 & 0 & 0 & 0 & 0 & 1 & 0 & 0 & 0 & 0 & 1 & 3 & 0\end{array}$ Definition - $0 \begin{array}{llllllllllllllllllllllllllll}0 & 0 & 0 & 0 & 22 & 0 & 0 & 0 & 0 & 0 & 0 & 0 & 0 & 0 & 0 & 0\end{array}$

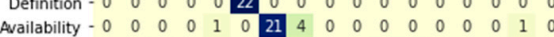
fault_tolerance $\begin{array}{rlllllllllllllllll}0 & 0 & 2 & 0 & 0 & 0 & 0 & 15 & 0 & 0 & 1 & 1 & 0 & 0 & 0 & 0 & 0\end{array}$

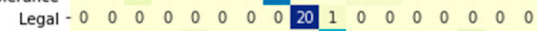
Look\&Feel - $0 \begin{array}{llllllllllllllllll}0 & 0 & 0 & 1 & 0 & 0 & 0 & 0 & 12 & 0 & 0 & 0 & 0 & 2 & 0 & 0\end{array}$

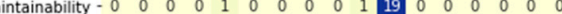

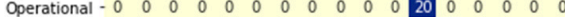
Performance - $0 \begin{array}{lllllllllllllllll}0 & 0 & 0 & 0 & 0 & 0 & 0 & 0 & 0 & 0 & 0 & 0 & 22 & 0 & 0 & 0 & 0\end{array}$

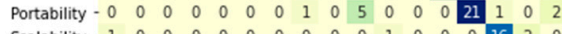

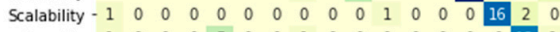

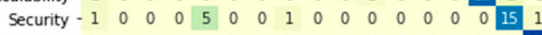

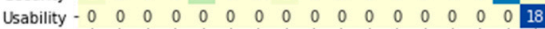

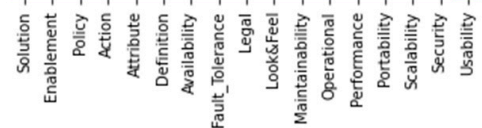

(a) BiLSTM

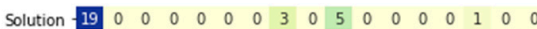

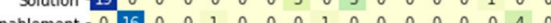

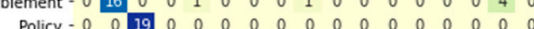

Policy - $0190 \begin{array}{llllllllllllll}1 & 0 & 0 & 0 & 0 & 0 & 0 & 0 & 0 & 0 & 0 & 0 & 0 & 0\end{array}$ Action - $0 \begin{array}{lllllllllllllllll}0 & 0 & 0 & 22 & 0 & 0 & 0 & 0 & 0 & 0 & 0 & 0 & 0 & 0 & 0 & 0\end{array}$ Attribute - $-0 \begin{array}{llllllllllllllllll}0 & 0 & 0 & 0 & 7 & 0 & 0 & 0 & 0 & 0 & 0 & 0 & 0 & 0 & 0 & 0 & 0\end{array}$ Definition - $0 \begin{array}{llllllllllllllllll} & 0 & 0 & 0 & 1 & \mathbf{2 2} & 0 & 0 & 0 & 1 & 0 & 0 & 0 & 0 & 0 & 3 & 0\end{array}$ Avallability - $0 \begin{array}{lllllllllllllllllll}0 & 0 & 0 & 0 & 1 & 0 & 20 & 0 & 0 & 0 & 1 & 0 & 0 & 0 & 0 & 2 & 0\end{array}$ Fault_tolerance - $0 \begin{array}{lllllllllllllllll}0 & 0 & 0 & 0 & 1 & 0 & 1 & 12 & 0 & 0 & 0 & 0 & 0 & 0 & 0 & 0 & 2\end{array}$

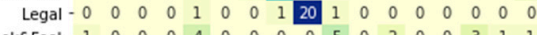

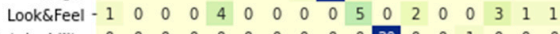
Maintainability - $0 \begin{array}{llllllllllllllll}0 & 0 & 0 & 0 & 0 & 0 & 0 & 0 & 0 & 20 & 0 & 0 & 1 & 0 & 0 & 0\end{array}$

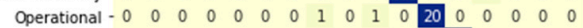

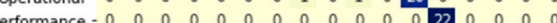
Portability - $0 \begin{array}{lllllllllllllllllll} & 0 & 0 & 0 & 0 & 0 & 0 & 0 & 0 & 0 & 0 & 0 & 0 & 20 & 0 & 0 & 1 & -5.0\end{array}$ $\begin{array}{lllllllllllllll}0 & 0 & 0 & 0\end{array}$ Security - $0 \begin{array}{cccccccccccccccc}5 & 2 & 0 & 0 & 0 & 0 & 0 & 0 & 2 & 0 & 0 & 0 & 0 & 1 & 10 & 0\end{array}$ Usability - $2 \begin{array}{llllllllllllllllllll}0 & 0 & 0 & 2 & 0 & 0 & 0 & 0 & 4 & 0 & 0 & 0 & 0 & 1 & 0 & 17\end{array}$

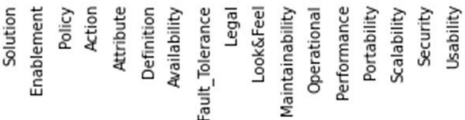

(c) GRU

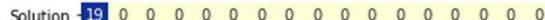
Enablement - $0 \begin{array}{llllllllllllllll}21 & 0 & 0 & 0 & 0 & 0 & 0 & 0 & 0 & 0 & 0 & 0 & 0 & 0 & 0 & 0\end{array}$ Policy - $0 \begin{array}{lllllllllllllllllll}0 & 18 & 0 & 0 & 0 & 0 & 0 & 0 & 0 & 0 & 0 & 0 & 0 & 0 & 0 & 0\end{array}$ Action - $0 \begin{array}{lllllllllllllllllllllllllll} & 0 & 0 & 0 & 0 & 0 & 0 & 0 & 0 & 0 & 0 & 0 & 0 & 0 & 0 & 0\end{array}$ Atribute - 0 Definition - 0

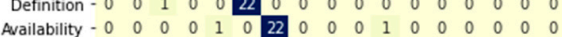

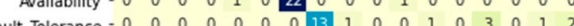
$\begin{array}{lllllllllllllllll} & & 0 & 0\end{array}$ Legal - $-0 \begin{array}{lllllllllllllllllllllll}0 & 0 & 0 & 0 & 0 & 0 & 0 & 0 & 20 & 1 & 0 & 0 & 0 & 0 & 0 & 0 & 0\end{array}$ Look\&Feel - $0 \begin{array}{llllllllllllllll}0 & 0 & 0 & 0 & 0 & 0 & 3 & 0 & 16 & 0 & 0 & 0 & 0 & 0 & 0 & 1\end{array}$

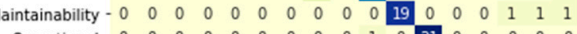

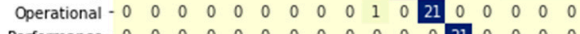
Performance - $0 \begin{array}{lllllllllllllllllllllll}0 & 0 & 0 & 0 & 0 & 0 & 0 & 0 & 0 & 0 & 0 & 0 & 21 & 0 & 0 & 0 & 0\end{array}$

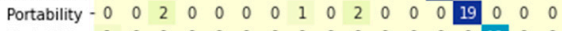

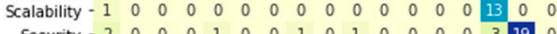

$\begin{array}{llllllllllllllllll}\text { Security - } 2 & 0 & 0 & 0 & 1 & 0 & 0 & 1 & 0 & 1 & 0 & 0 & 0 & 0 & 3 & 19 & 0 \\ \text { Usability - } & 0 & 0 & 0 & 1 & 0 & 0 & 3 & 0 & 0 & 0 & 0 & 0 & 0 & 0 & 0 & 17\end{array}$

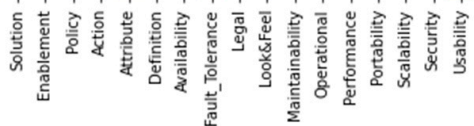

(e) Mean ensemble

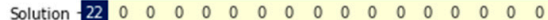

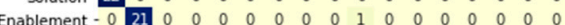
Policy - 0 Action - $000 \begin{array}{lllllllllllllllll}22 & 0 & 0 & 0 & 0 & 0 & 0 & 0 & 0 & 0 & 0 & 0 & 1 & 0\end{array}$ Atribute - $0 \begin{array}{llllllllllllllll}0 & 0 & 0 & 15 & 0 & 0 & 1 & 0 & 1 & 0 & 0 & 0 & 0 & 1 & 0 & 0\end{array}$

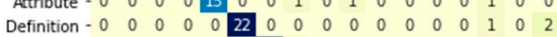
Availability - $0 \begin{array}{llllllllllllllll}0 & 0 & 0 & 0 & 0 & 21 & 1 & 0 & 0 & 0 & 0 & 0 & 0 & 0 & 0 & 0\end{array}$

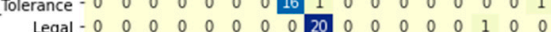

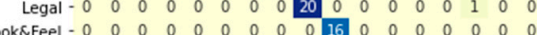

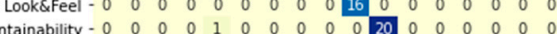
Maintainability - $-0 \begin{array}{lllllllllllllllll}0 & 0 & 0 & 1 & 0 & 0 & 0 & 0 & 0 & 0 & 0 & 0 & 0 & 0 & 0 & 0\end{array}$ Performance - $0 \begin{array}{lllllllllllllllll} & 0 & 0 & 0 & 0 & 0 & 0 & 0 & 0 & 0 & 0 & 0 & 22 & 0 & 0 & 0 & 0\end{array}$

Portability - $0 \begin{array}{lllllllllllllllll}0 & 0 & 0 & 0 & 0 & 0 & 0 & 0 & 0 & 2 & 0 & 0 & 0 & 21 & 0 & 0 & 1\end{array}$

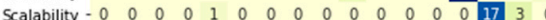

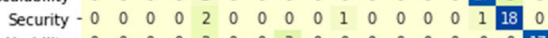

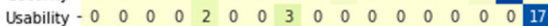

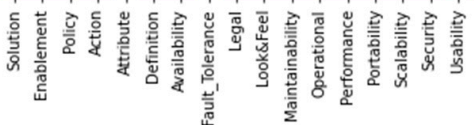
(b) $\mathrm{CNN}$

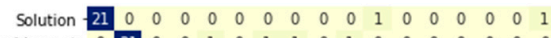
Enablement - $0 \begin{array}{llllllllllllllll}21 & 0 & 0 & 1 & 0 & 1 & 1 & 0 & 1 & 0 & 0 & 0 & 0 & 0 & 0 & 0\end{array}$ Policy - $0 \begin{array}{llllllllllllllll}21 & 0 & 0 & 0 & 0 & 0 & 0 & 0 & 0 & 0 & 0 & 0 & 1 & 0 & 0\end{array}$

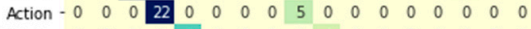

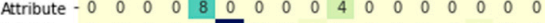
Definition - $0 \begin{array}{lllllllllllllllll}0 & 0 & 0 & 0 & 22 & 0 & 0 & 1 & 0 & 0 & 0 & 0 & 0 & 1 & 0 & 0\end{array}$

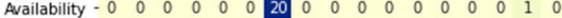
Fault_Tolerance - $0 \begin{array}{lllllllllllllllll}0 & 0 & 0 & 0 & 6 & 0 & 0 & 15 & 0 & 2 & 2 & 1 & 0 & 0 & 2 & 0 & 2\end{array}$

Legal-0 $00 \begin{array}{lllllllllllllll}0 & 0 & 0 & 0 & 0 & 0 & 15 & 0 & 0 & 0 & 0 & 0 & 0 & 0 & 0\end{array}$

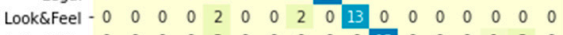

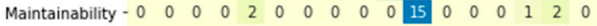

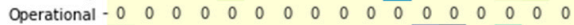

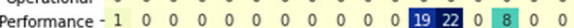
Portability - $0 \begin{array}{lllllllllllllllllllllll}0 & 0 & 0 & 2 & 0 & 0 & 0 & 0 & 1 & 0 & 0 & 0 & 19 & 0 & 3 & 0\end{array}$ Scalability - $00 \begin{array}{lllllllllllllllllllll}0 & 0 & 0 & 0 & 0 & 0 & 0 & 0 & 0 & 0 & 0 & 0 & 0 & 7 & 0 & 0\end{array}$ $\begin{array}{ccccccccccccccccc}\text { Security }-0 & 0 & 0 & 0 & 0 & 0 & 0 & 3 & 0 & 0 & 0 & 2 & 0 & 0 & 1 & 12 & 0\end{array}$ Usability - $0 \begin{array}{lllllllllllllllllllll}0 & 0 & 0 & 0 & 0 & 0 & 0 & 0 & 0 & 0 & 3 & 0 & 0 & 2 & 0 & 4 & 18\end{array}$

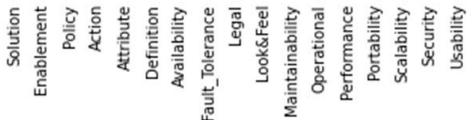

(d) LSTM

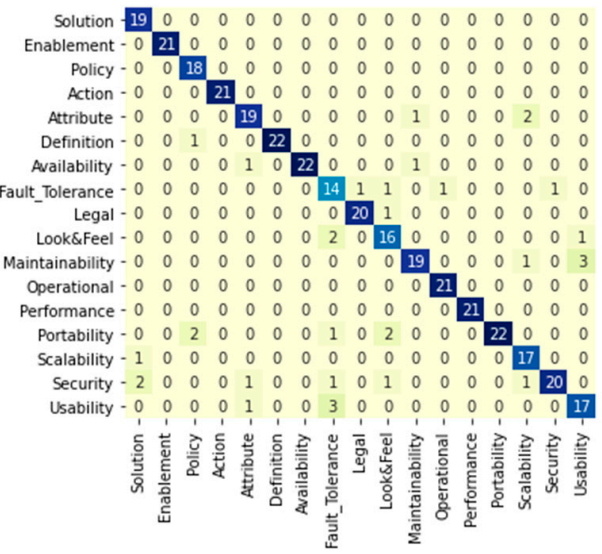

(f) Accuracy as a weight ensemble

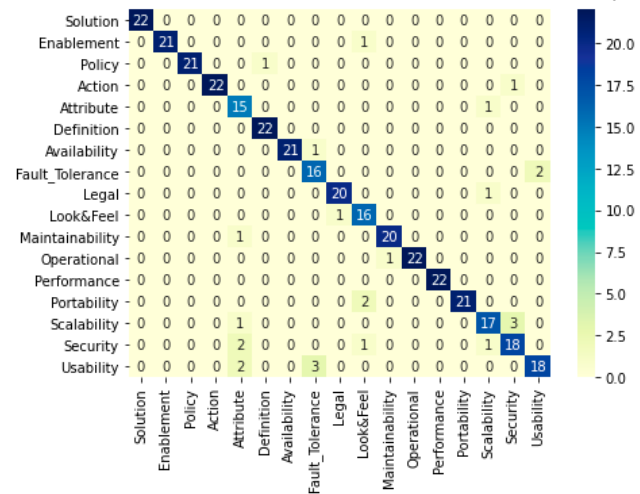

(g) Accuracy per class as a weight ensemble

Figure 6. One-phase system confusion matrices for the DL classifiers. 


\subsection{Two-Phase Classification System}

\subsubsection{Binary Classification of SRs into an FR or NFR Phase}

\section{A. Training}

This system classifies SRs into FRs or NFRs as the first phase, and the preprocessed and binary-classified SRs are then passed to the second phase for multi-class classification. The validation dataset was used to ensure that there was no overfitting in each base DL classifier. The hyperparameters were left as default, and some were justified to values that help to avoid overfitting. The Adam optimizer was used with a 0.001 default learning rate. The suitable number of epochs was 30 with a batch size of 50. Dropout was justified to 0.2 for all classifiers. The architecture of each base DL model is summarized in Tables 11-14.

Table 11. Two-phase system: architecture of the BiLSTM model in the binary classification phase.

\begin{tabular}{ccc}
\hline Layer & Output Shape & Number of Parameters \\
\hline Embedding_68 & (None, None, 46) & 60,858 \\
Spatial_dropout1d_40 & (None, None, 46) & 0 \\
Bidirectional_40 & (None, 92) & 34,224 \\
Dense_156 & (None, 46) & 4278 \\
Dropout_99 & (None, 46) & 0 \\
Dense_157 & (None, 46) & 2162 \\
Dropout_100 & (None, 46) & 0 \\
Dense_158 & (None, 3) & 141 \\
Activation_40 & (None, 3) & 0 \\
\hline
\end{tabular}

Table 12. Two-phase system: architecture of the CNN model in the binary classification phase.

\begin{tabular}{ccc}
\hline Layer & Output Shape & Number of Parameters \\
\hline Embedding_69 & (None, None, 46) & 60,858 \\
Dropout_101 & (None, None, 46) & 0 \\
Conv1d_11 & (None, None, 46) & 6394 \\
Global_max_pooling1d_11 & (None, 46) & 0 \\
Dense_159 & (None, 128) & 6016 \\
Dropout_102 & (None, 128) & 0 \\
Dense_160 & (None, 2) & 258 \\
\hline
\end{tabular}

Table 13. Two-phase system: architecture of the GRU model in the binary classification phase.

\begin{tabular}{ccc}
\hline Layer & Output Shape & Number of Parameters \\
\hline Embedding_70 & (None, None, 46) & 60,858 \\
Gru_19 & (None, None, 46) & 12,834 \\
Gru_20 & (None, 32) & 7584 \\
Dense_161 & (None, 2) & 66 \\
\hline
\end{tabular}

Table 14. Two-phase system: architecture of the LSTM model in the binary classification phase.

\begin{tabular}{ccc}
\hline Layer & Output Shape & Number of Parameters \\
\hline Embedding_71 & (None, None, 46) & 60,858 \\
Lstm_59 & (None, None, 46) & 17,112 \\
Lstm_60 & (None, 32) & 10,112 \\
Dense_162 & (None, 2) & 66 \\
\hline
\end{tabular}

B. Test Results and discussion

Table 15 displays the performance attributes of each DL model and ensemble method applied to the PROMISE dataset during the binary classification phase of SRs. The DL models were able to reach close results of $94.49 \%, 93.22 \%, 93.22 \%$, and $92.37 \%$ by BiLSTM, 
LSTM, CNN, and GRU, respectively. However, in terms of the ensemble methods, the accuracy as a weight and accuracy per class as a weight were able to enhance the accuracy by achieving $94.9 \%$ and $95.7 \%$, respectively. The other performance evaluation attributes achieved 0.96 by using accuracy per class as a weight ensemble method. Figure 7 shows the confusion matrices for all of the DL models and ensemble methods and clarifies that the best prediction accuracy was achieved by accuracy per class as a weight ensemble method.

Table 15. Two-phase system evaluation parameters for the DL models in the binary classification phase.

\begin{tabular}{ccccc}
\hline DL Models & $\begin{array}{c}\text { Accuracy } \\
\mathbf{( \% )}\end{array}$ & Precision & Recall & F1 Score \\
\hline BiLSTM & 95.00 & 0.94 & 0.95 & 0.94 \\
LSTM & 93.22 & 0.93 & 0.93 & 0.93 \\
CNN & 93.22 & 0.93 & 0.94 & 0.93 \\
GRU & 92.37 & 0.92 & 0.92 & 0.92 \\
Mean ensemble & 94.4 & 0.94 & 0.95 & 0.94 \\
Accuracy as a weight ensemble & 94.9 & 0.95 & 0.95 & 0.95 \\
Accuracy per class as a weight ensemble & 96 & 0.96 & 0.96 & 0.96 \\
\hline
\end{tabular}

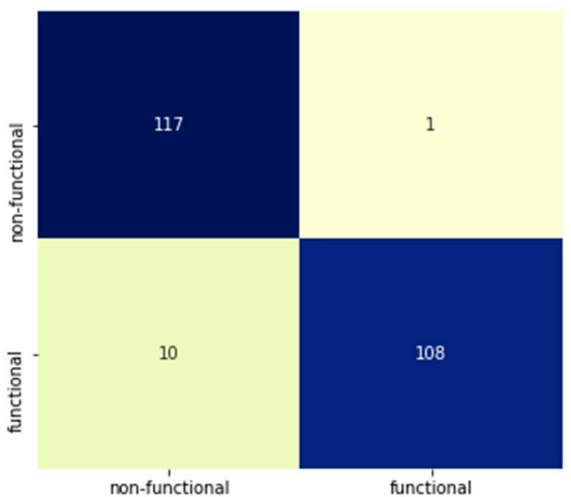

(a) BiLSTM

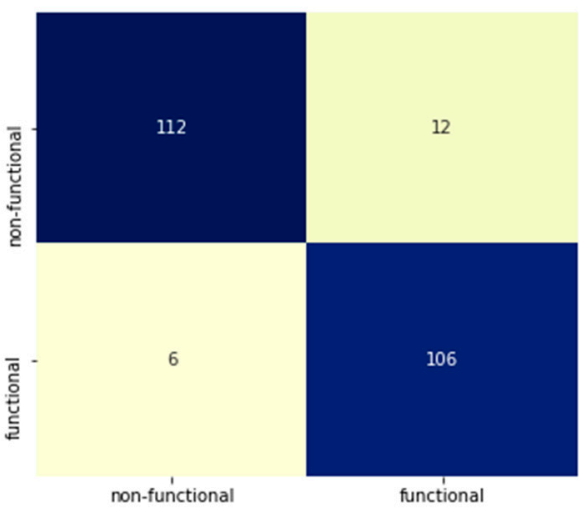

(c) GRU
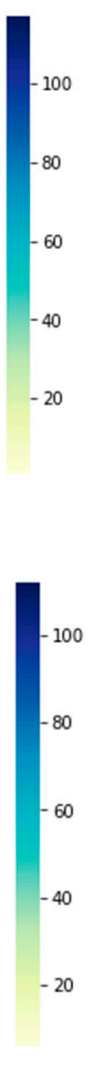

20

non-functional
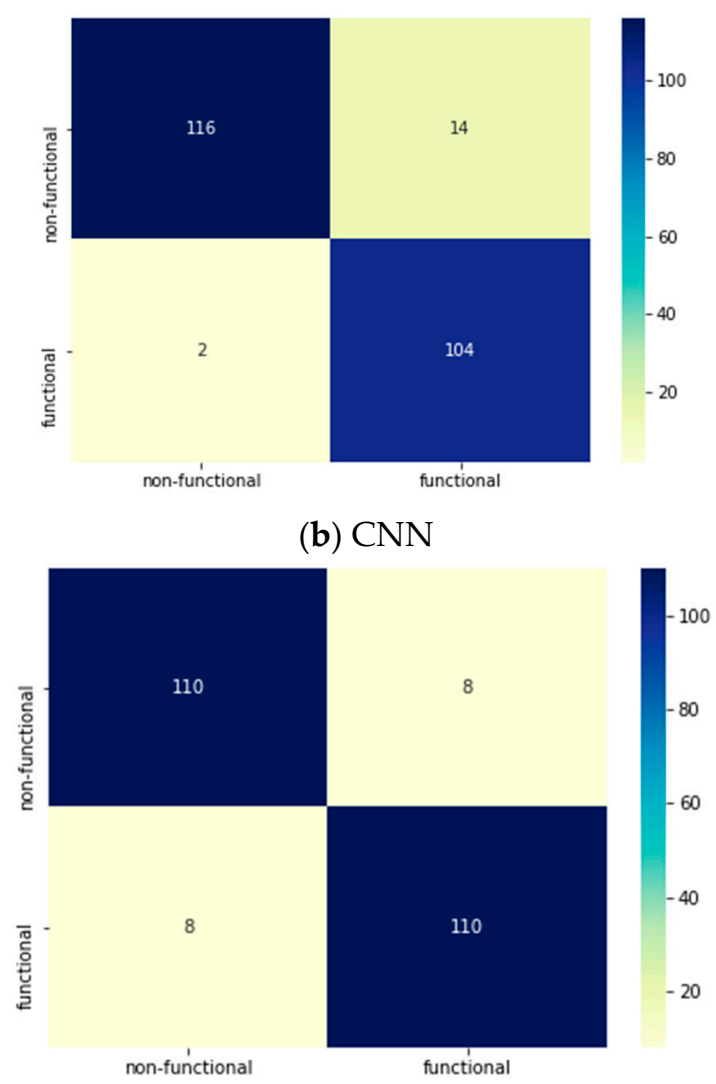

(b) $\mathrm{CNN}$

(d) LSTM

Figure 7. Cont. 


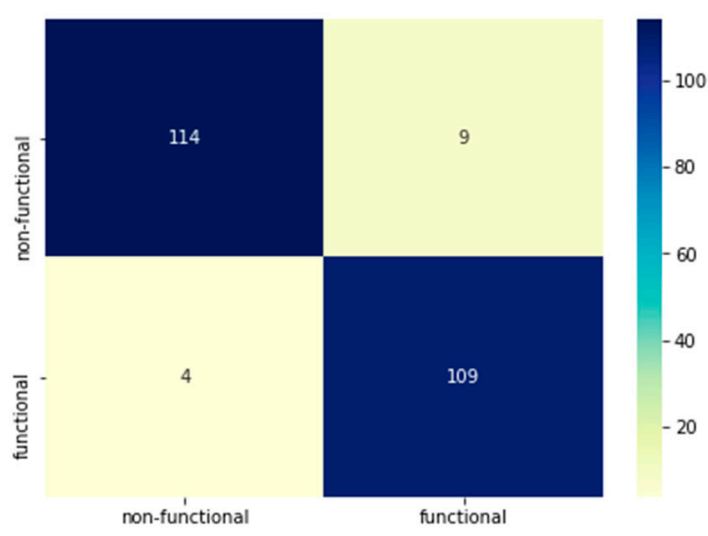

(e) Mean ensemble

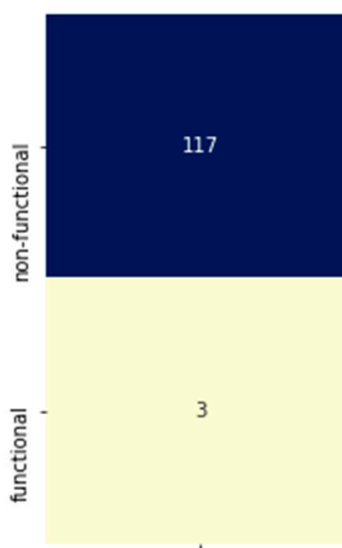

non-functional

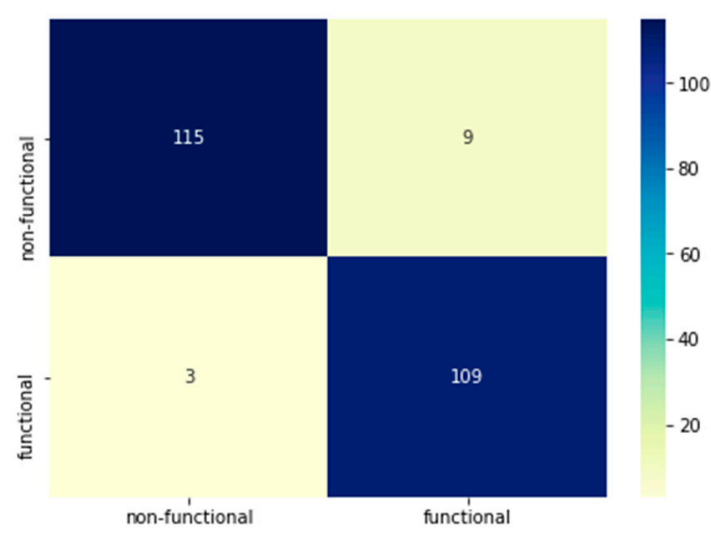

(f) Accuracy as a weight ensemble

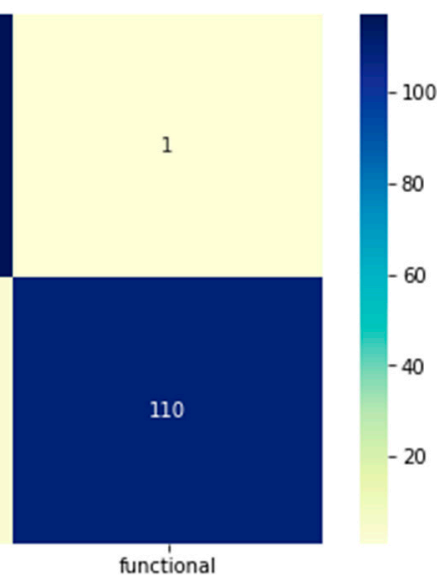

functional

(g) Accuracy per class as a weight ensemble

Figure 7. Two-phase system: Confusion matrices for the DL models in the binary classification phase.

\subsubsection{Multi-Class Classification Phase of NFRs and FRs into 17 Classes}

\section{A. Training}

Training the model to handle FRs and NFRs requires different parameters to be tuned and justified to achieve acceptable results, as the model should be trained to modify itself to search FR classes if the input from the first phase (binary classification) is FRs and to search in the NFR classes if the input from the first phase is NFRs. Tables 16-19 summarize the parameters of the base DL models in the training phase for FRs, and Tables 20-23 summarize the parameters of the base DL models in the training phase of NFRs.

Table 16. Two-phase system: architecture of the BiLSTM model in the FR multi-class classification phase.

\begin{tabular}{ccc}
\hline Layer & Output Shape & Number of Parameters \\
\hline Embedding_8 & (None, None, 46) & 28,106 \\
Spatial_dropout1d_3 & (None, None, 46) & 0 \\
Bidirectional_3 & (None, 92) & 34,224 \\
Dense_14 & (None, 46) & 4278 \\
Dropout_9 & (None, 46) & 0 \\
Dense_15 & (None, 46) & 2162 \\
Dropout_10 & (None, 46) & 0 \\
Dense_16 & (None, 6) & 282 \\
Activation_3 & (None, 6) & 0 \\
\hline
\end{tabular}


Table 17. Two-phase system: architecture of the CNN model in the FR multi-class classification phase.

\begin{tabular}{ccc}
\hline Layer & Output Shape & Number of Parameters \\
\hline Embedding_9 & (None, None, 46) & 28,106 \\
Dropout1d_11 & (None, None, 46) & 0 \\
Conv1d_3 & (None, None, 46) & 6394 \\
Global_max_pooling1d_3 & (None, 46) & 0 \\
Dense_17 & (None, 128) & 6016 \\
Dropout_12 & (None, 128) & 0 \\
Dense_18 & (None, 6) & 774 \\
\hline
\end{tabular}

Table 18. Two-phase system: architecture of the GRU model in the FR multi-class classification phase.

\begin{tabular}{ccc}
\hline Layer & Output Shape & Number of Parameters \\
\hline Embedding_10 & (None, None, 46) & 28,106 \\
GRU_5 & (None, None, 46) & 12,834 \\
GRU_6 & (None, 32) & 7584 \\
Dense_19 & (None, 6) & 198 \\
\hline
\end{tabular}

Table 19. Two-phase system: architecture of the LSTM model in the FR multi-class classification phase.

\begin{tabular}{ccc}
\hline Layer & Output Shape & Number of Parameters \\
\hline Embedding_11 & (None, None, 46) & 28,106 \\
Lstm_6 & (None, None, 46) & 17,112 \\
Lstm_7 & (None, 32) & 10,112 \\
Dense_20 & (None, 6) & 198 \\
\hline
\end{tabular}

Table 20. Two-phase system: architecture of the BiLSTM model in the NFR multi-class classification phase.

\begin{tabular}{ccc}
\hline Layer & Output Shape & Number of Parameters \\
\hline Embedding_4 & (None, None, 46) & 53,958 \\
Spatial_dropout1d_4 & (None, None, 46) & 0 \\
Bidirectional_4 & (None, 92) & 34,224 \\
Dense_21 & (None, 46) & 4278 \\
Dropout_13 & (None, 46) & 0 \\
Dense_22 & (None, 46) & 2162 \\
Dropout_14 & (None, 46) & 0 \\
Dense_23 & (None, 11) & 517 \\
Activation_4 & (None, 11) & 0 \\
\hline
\end{tabular}

Table 21. Two-phase system: architecture of the CNN model in the NFR multi-class classification phase.

\begin{tabular}{ccc}
\hline Layer & Output Shape & Number of Parameters \\
\hline Embedding_13 & (None, None, 46) & 53,958 \\
Dropout_15 & (None, None, 46) & 0 \\
Conv1d_4 & (None, None, 46) & 6394 \\
Global_max_pooling1d_4 & (None, 46) & 0 \\
Dense_24 & (None, 128) & 6016 \\
Dropout_16 & (None, 128) & 0 \\
Dense_25 & (None, 11) & 1419 \\
\hline
\end{tabular}


Table 22. Two-phase system: architecture of the GRU model in the NFR multi-class classification phase.

\begin{tabular}{ccc}
\hline Layer & Output Shape & Number of Parameters \\
\hline Embedding_14 & (None, None, 46) & 53,958 \\
GRU_7 & (None, None, 46) & 12,834 \\
GRU_8 & (None, 32) & 7584 \\
Dense_26 & (None, 11) & 363 \\
\hline
\end{tabular}

Table 23. Two-phase system: architecture of the LSTM model in the NFR multi-class classification phase.

\begin{tabular}{ccc}
\hline Layer & Output Shape & Number of Parameters \\
\hline Embedding_15 & (None, None, 46) & 53,958 \\
Lstm_9 & (None, None, 46) & 17,112 \\
Lstm_10 & (None, 32) & 10,112 \\
Dense_27 & (None, 11) & 363 \\
\hline
\end{tabular}

\section{B. Test Results and discussion}

Table 24 clarifies the results of all of the used DL models and ensemble methods that have been applied to classify FRs from the PROMISE dataset after being preprocessed and binary classified by the same model in the previous phase. The base DL models performed successfully in the case of BiLSTM and CNN, as they achieved accuracies of $96.8 \%$ and $96.0 \%$, respectively. However, the LSTM and GRU models only achieved accuracies of $78.91 \%$ and $58.59 \%$, respectively, indicating lower performance than the other DL models. The ensemble methods achieved equal accuracy percentages as BiLSTM model (96.8\%). All confusion matrices for all DL models and ensemble methods are displayed in Figure 8 . It can be clearly seen that the best predictions for all FR classes were achieved by accuracy per class as a weight ensemble method.

Table 24. Two-phase system evaluation parameters of the DL classifiers for FRs in the multi-class classification phase.

\begin{tabular}{ccccc}
\hline DL Models & $\begin{array}{c}\text { Accuracy } \\
\mathbf{( \% )}\end{array}$ & Precision & Recall & F1 Score \\
\hline BiLSTM & 96.8 & 0.97 & 0.97 & 0.97 \\
LSTM & 78.91 & 0.79 & 0.79 & 0.79 \\
CNN & 96.0 & 0.96 & 0.96 & 0.96 \\
GRU & 58.59 & 0.59 & 0.59 & 0.59 \\
Mean ensemble & 96.8 & 0.97 & 0.97 & 0.97 \\
Accuracy as a weight ensemble & 96.8 & 0.97 & 0.97 & 0.97 \\
Accuracy per class as a weight ensemble & 98 & 0.98 & 0.98 & 0.98 \\
\hline
\end{tabular}

Table 25 displays the performance of all the applied classifiers: DL models and ensemble methods in the NFR multi-class classification phase, which comes after the binary phase. In terms of the base DL models, BiLSTM and CNN achieved accuracies of approximately $83 \%$, while LSTM and GRU achieved accuracies of $63 \%$ and $56 \%$, respectively. All ensemble methods successfully improved the accuracy. However, the best accuracy was achieved by accuracy per class ensemble method (86.5\%). The recall and F1 score were 0.86 and 0.87 , respectively, but the precision was 0.89 . Moreover, Figure 9 displays the predictions of each DL model and ensemble method with a clear indication that accuracy per class as a weight ensemble method has the maximum prediction values among the others. 


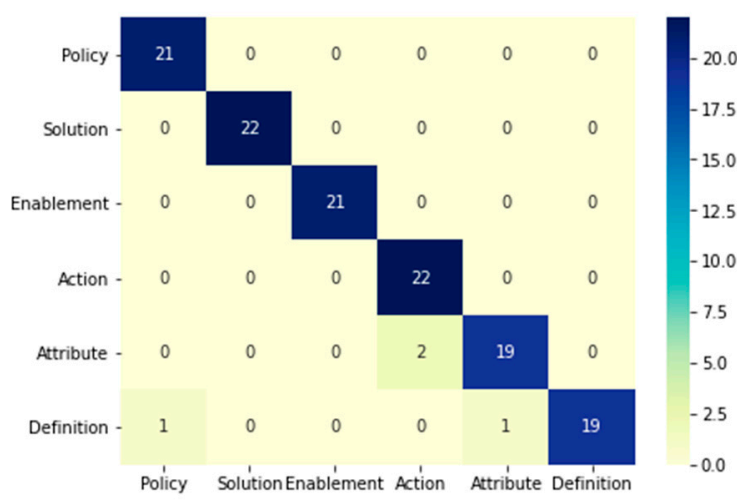

(a) BiLSTM

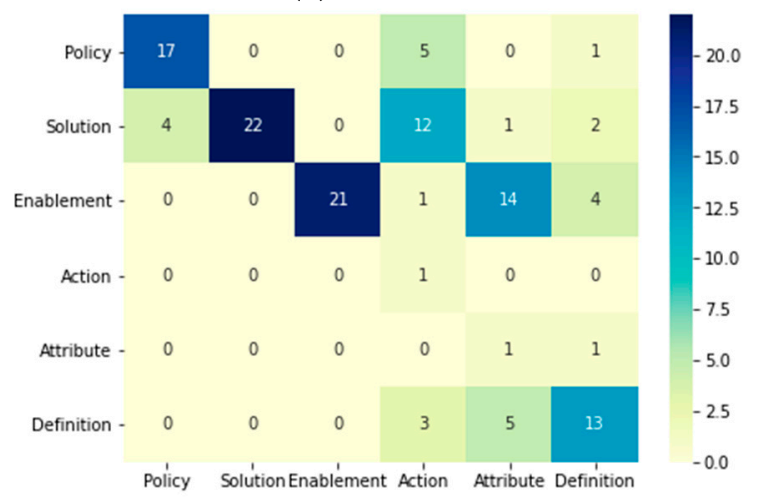

(c) GRU

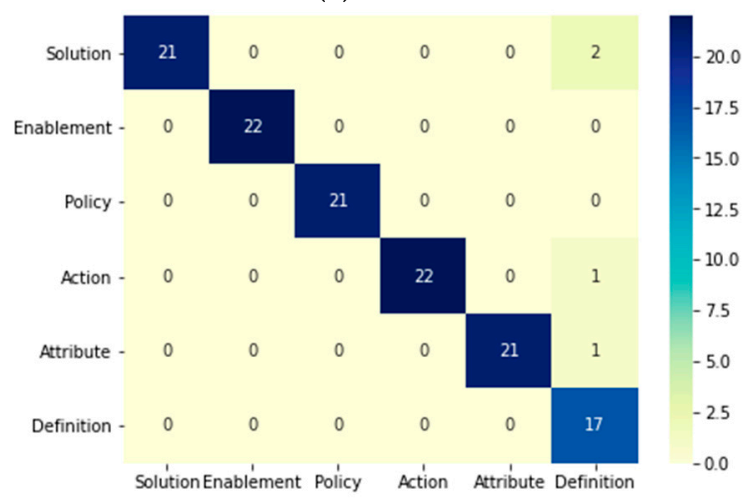

(e) Mean ensemble

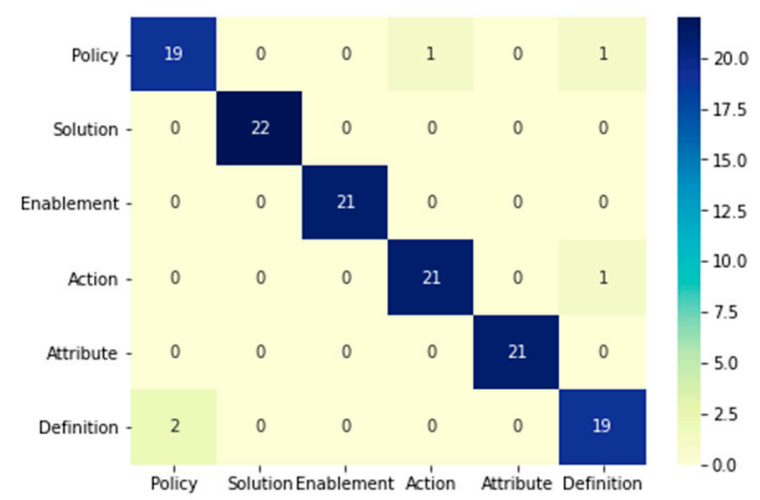

(b) CNN

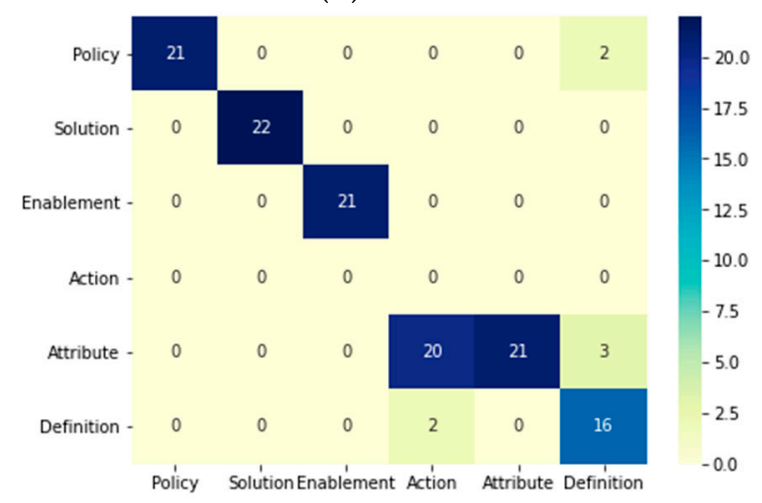

(d) LSTM

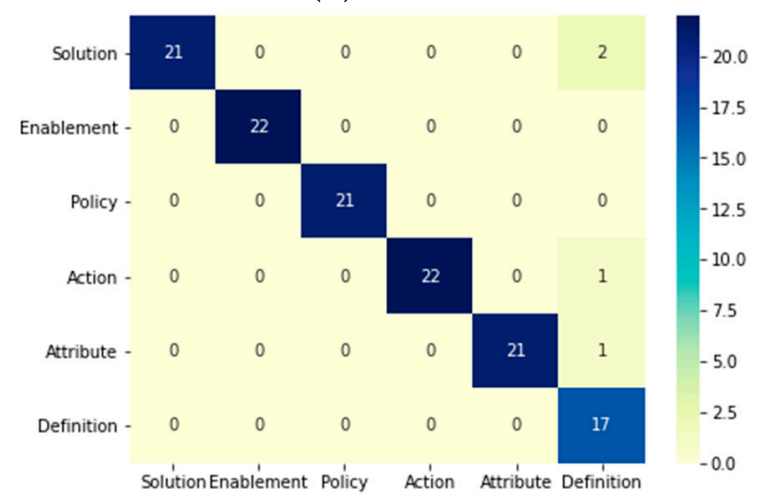

(f) Accuracy as a weight ensemble

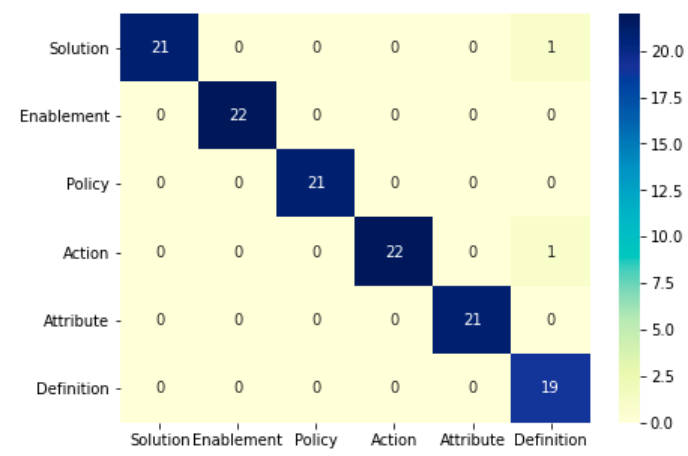

(g) Accuracy per class as a weight ensemble

Figure 8. Two-phases system: confusion matrices of the FR DL classifiers in the multi-class classification phase. 
Table 25. Two-phase system: evaluation parameters for the NFR DL classifiers in the multi-class classification phase.

\begin{tabular}{ccccc}
\hline DL Models & $\begin{array}{c}\text { Accuracy } \\
\text { (\%) }\end{array}$ & Precision & Recall & F1 Score \\
\hline BiLSTM & 83.7 & 0.84 & 0.85 & 0.84 \\
LSTM & 63.0 & 0.64 & 0.63 & 0.64 \\
CNN & 83.3 & 0.83 & 0.83 & 0.83 \\
GRU & 56.0 & 0.57 & 0.56 & 0.55 \\
Mean ensemble & 85.0 & 0.85 & 0.84 & 0.85 \\
Accuracy as a weight ensemble & 85.1 & 0.85 & 0.85 & 0.85 \\
Accuracy per class as a weight & 86.5 & 0.89 & 0.86 & 0.87 \\
\hline
\end{tabular}

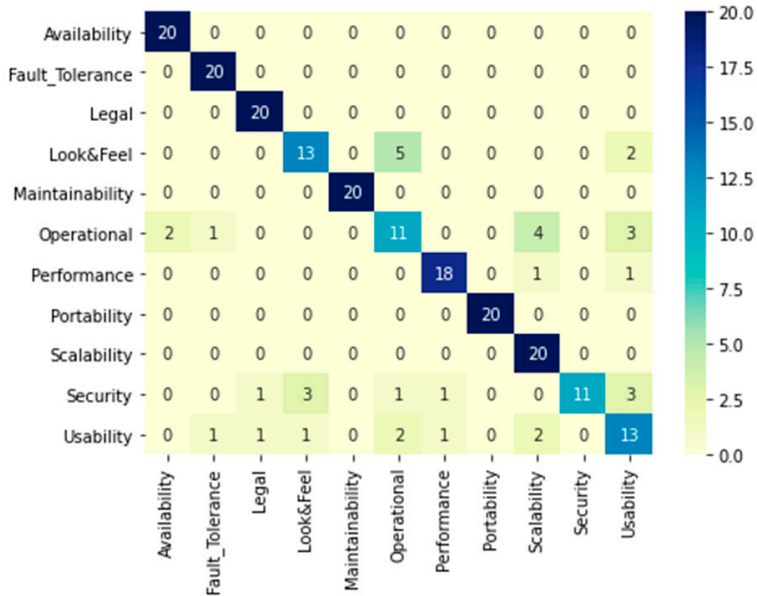

(a) BiLSTM

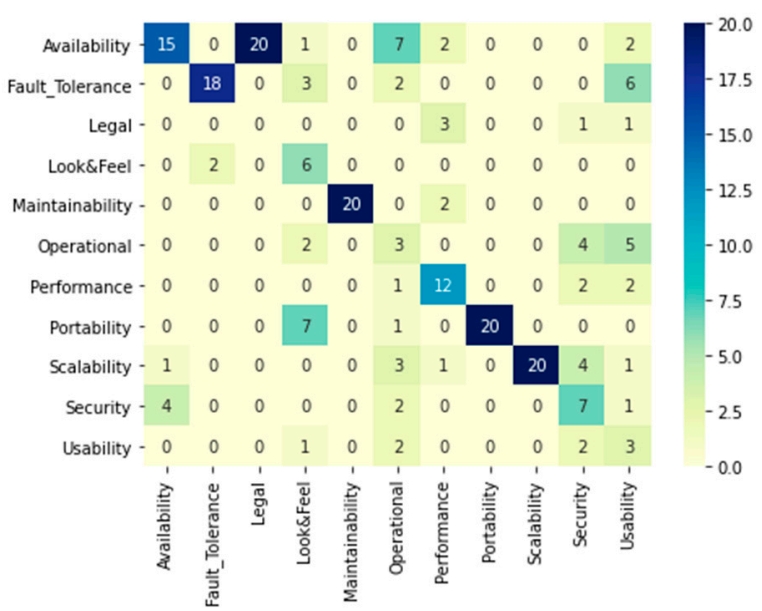

(c) GRU

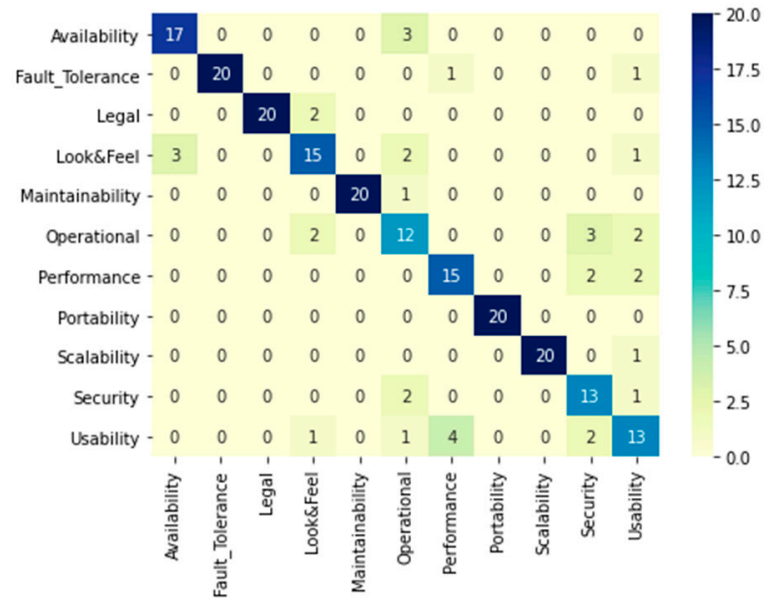

(b) $\mathrm{CNN}$

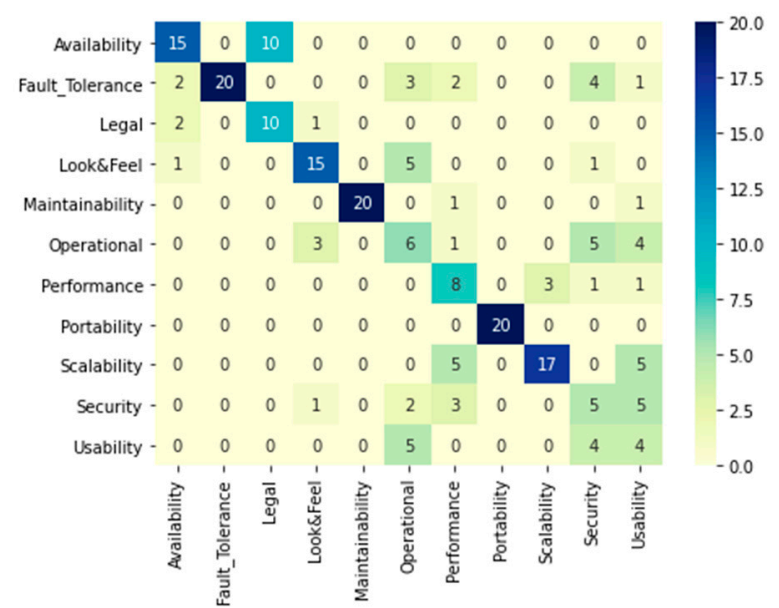

(d) LSTM

Figure 9. Cont. 


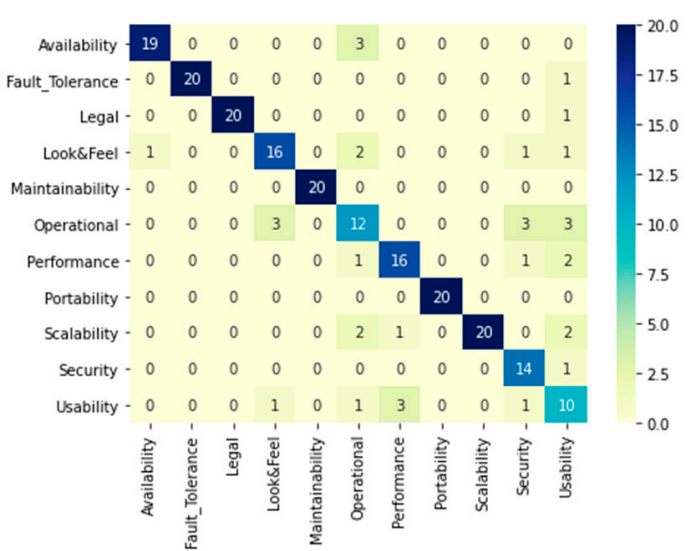

(e) Mean ensemble

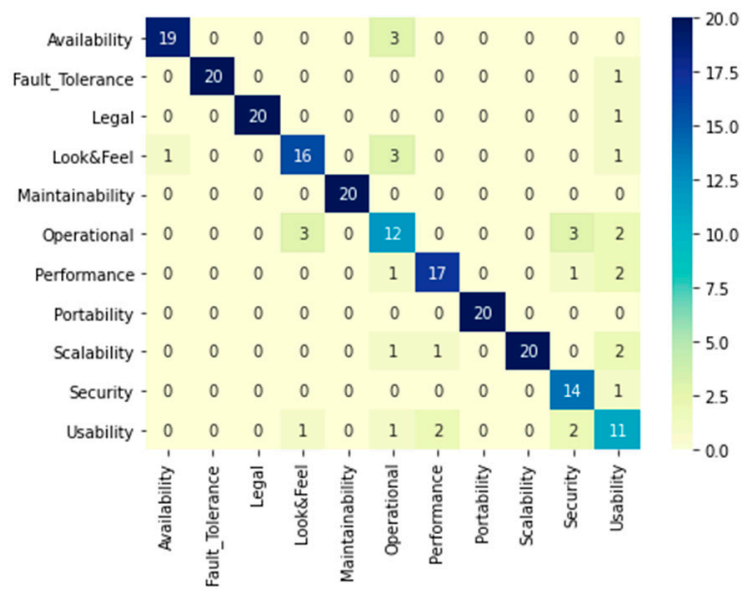

(f) Accuracy as a weight ensemble

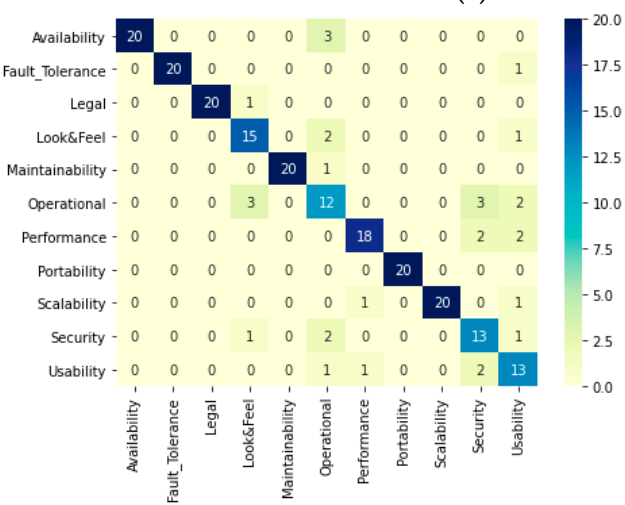

(g) Accuracy per class as a weight ensemble

Figure 9. Two-phase system: Confusion matrices of the NFR DL classifiers in the multi-class classification phase.

\subsubsection{All Together Two-Phase Classification System}

Table 26 displays the final results of the evaluation parameters for the two-phase classification system all together. All DL models and ensemble methods performed better when using the two-phase classification system, as the binary phase facilitates the multiclass classification phase because it carries out the preprocessing and informs each model to justify itself to either focus on FR or NFR classes. Better evaluation parameters were achieved, but the accuracy per class as a weight ensemble recorded the best parameter values among the other DL models and ensemble methods, and even compared to itself in one-phase classification system, as it scored a $93.4 \%$ accuracy, a 0.94 precision, a 0.94 recall, and a 0.93 F1 score.

Table 26. Two-phase classification system: Evaluation parameters of all phases together.

\begin{tabular}{ccccc}
\hline DL Models & $\begin{array}{c}\text { Average } \\
\text { Accuracy } \\
\mathbf{( \% )}\end{array}$ & $\begin{array}{c}\text { Average } \\
\text { Precision }\end{array}$ & $\begin{array}{c}\text { Average } \\
\text { Recall }\end{array}$ & $\begin{array}{c}\text { Average F1 } \\
\text { Score }\end{array}$ \\
\hline BiLSTM & 91.66 & 0.91 & 0.92 & 0.91 \\
LSTM & 78.37 & 0.78 & 0.78 & 0.78 \\
CNN & 90.84 & 0.90 & 0.91 & 0.90 \\
GRU & 68.98 & 0.69 & 0.69 & 0.68 \\
Mean ensemble & 92.06 & 0.92 & 0.92 & 0.92 \\
Accuracy as a weight ensemble & 92.26 & 0.92 & 0.92 & 0.92 \\
Accuracy per class as a weight & 93.4 & 0.94 & 0.93 & 0.93 \\
\hline
\end{tabular}




\section{Comparative Analysis}

In order to prove that the two-phase classification system using the ensemble method based on DL models has an impact on the field and shows the best performance among the existing state-of-the-art methodologies, it was compared experimentally with other approaches applied on the same dataset (PROMISE). Table 27 summarizes the results of previous studies that have applied their suggested methodology using ML or DL on PROMISE dataset and the proposed system. In [7], the authors achieved a 0.80 precision in classifying SRs into 11 classes of NFRs or FRs if it does not belong to one of the eleven classes using the CNN model. However, in [19], the authors started by binary classification of SRs, recording a $0.94 \mathrm{~F} 1$ score. Then, NFRs were classified using a smaller number of classes, as used in [20], achieving a $0.91 \mathrm{~F} 1$ score, but NFRs were also classified into securityrelated or non-security-related, with a $0.77 \mathrm{~F} 1$ score. In [17], the authors classified NFRs into 11 classes, scoring a 76\% accuracy. Only [20] provided a complete classification system. SRs were classified into FRs or NFRs with an average F1 score of $91.5 \%$. Then, NFRs were classified into four classes only: Usability, security, operational, and performance, with a $76 \%$ F1 score. FRs were also classified into FRs or data behavior, with a 92\% F1 score. Although a complete system was presented, the proposed classification systems herein outperform this system. Moreover, it only classifies NFRs into four classes, while the proposed classification system here classifies NFRs into 11 classes. In addition, using the previously mentioned system, FRs are classified into two classes, but our proposed system classifies FRs into six classes. The main comparison attribute is the performance, as the proposed one-phase classification system achieved a $92.56 \%$ accuracy, which has not been achieved before. Moreover, the proposed two-phase classification system achieved a $95.7 \%$ accuracy in the binary phase, which is the highest among all previous studies' results in binary classification. In the second phase of the multi-class classification, it scored a 93.4\% accuracy. This is clear confirmation that the proposed systems are better than others, with the two-phase classification system being better than the one-phase classification system.

Table 27. Comparison of the proposed system with other state-of-the-art systems.

\begin{tabular}{|c|c|c|c|c|c|}
\hline \multirow{2}{*}{$\begin{array}{c}\text { Reference \# } \\
\text { Year }\end{array}$} & \multicolumn{2}{|c|}{ Classification } & \multirow{2}{*}{ Methodology } & \multirow{2}{*}{ Dataset } & \multirow{2}{*}{ Results } \\
\hline & Input & Classes & & & \\
\hline \multicolumn{6}{|c|}{ 1. Classifying NFRs into Multi-Classes } \\
\hline [7] 2017 & SRs & $\begin{array}{c}\text { Functional Availability } \\
\text { Legal } \\
\text { Look and feel } \\
\text { Maintainability Operational } \\
\text { Performance Scalability } \\
\text { Security } \\
\text { Usability } \\
\text { Fault tolerance Portability }\end{array}$ & $\mathrm{CNN}$ & PROMISE corpus & $\begin{array}{l}\text { Precession: } 0.80 \\
\text { Recall: } 0.785 \\
\text { F measure: } 0.77\end{array}$ \\
\hline $\begin{array}{l}\text { [18] } 2018 \\
\text { 1. SR } \\
\text { 2. NFRs } \\
\text { 3. NFRs }\end{array}$ & $\begin{array}{c}\text { 1. FRs } \\
\text { NFRs } \\
\text { 2. Availability } \\
\text { Legal } \\
\text { Maintainability } \\
\text { Operational } \\
\text { Performance } \\
\text { Scalability } \\
\text { Look and feel } \\
\text { Security } \\
\text { Usability } \\
\text { 3. Security } \\
\text { Non-security }\end{array}$ & $\mathrm{CNN}$ & PROM corpus & \multicolumn{2}{|c|}{$\begin{array}{l}\text { 1. F1 score: } 0.945 \\
\text { 2. F1 score: } 0.911 \\
\text { 3. F1 score: } 0.772\end{array}$} \\
\hline [17] 2019 & SRs & $\begin{array}{c}\text { FR } \\
\text { Availability } \\
\text { Legal } \\
\text { Look and feel } \\
\text { Maintainability } \\
\text { Operational } \\
\text { Performance } \\
\text { Scalability } \\
\text { Security } \\
\text { Usability } \\
\text { Fault tolerance } \\
\text { Portability }\end{array}$ & $\begin{array}{c}\text { Multinomial naïve } \\
\text { Bayes (MNB) } \\
\text { Gaussian naïve Bayes } \\
\text { (GNB) } \\
\text { Bernoulli naïve Bayes } \\
\text { (BNB) } \\
\text { K-nearest neighbor } \\
\text { (KNN) } \\
\text { Support vector machine } \\
\text { (SVM) } \\
\text { Stochastic gradient } \\
\text { descent SVM (SGD } \\
\text { SVM) } \\
\text { Decision tree (Dtree) }\end{array}$ & PROMISE dataset & $\begin{array}{c}\text { Precision: } 0.66 \\
\text { Recall: } 0.61 \\
\text { F1 score: } 0.61 \\
\text { Accuracy: } 0.76 \\
\text { Using the SGD SVM } \\
\text { classifier and TF-IDF for } \\
\text { feature extraction }\end{array}$ \\
\hline
\end{tabular}


Table 27. Cont.

\begin{tabular}{|c|c|c|c|c|c|}
\hline \multirow{2}{*}{$\begin{array}{c}\text { Reference \# } \\
\text { Year }\end{array}$} & \multicolumn{2}{|c|}{ Classification } & \multirow{2}{*}{ Methodology } & \multirow{2}{*}{ Dataset } & \multirow{2}{*}{ Results } \\
\hline & Input & Classes & & & \\
\hline \multicolumn{6}{|c|}{ 2. Complete System (classifying NFRs and FRs into Multi-Classes) } \\
\hline [20] 2020 & $\begin{array}{l}\text { 1. SRs } \\
\text { 2. NFRs } \\
\text { 3. FRs }\end{array}$ & $\begin{array}{l}\text { 1. FRs-NFRs } \\
\text { 2. Usability } \\
\text { Security } \\
\text { Operational } \\
\text { Performance } \\
\text { 3. ions } \\
\text { Data behavior }\end{array}$ & $\begin{array}{c}\text { Fine-tuned BERT } \\
\text { (NoRBERT) }\end{array}$ & PROMISE & $\begin{array}{l}\text { 1. F1 Score: } 90 \% \text { FRs } \\
\text { and } 93 \% \text { NFRs } \\
\text { 2. F1 Score: } 76 \% \\
\text { 3. F1 Score: } 92 \%\end{array}$ \\
\hline $\begin{array}{c}2021 \\
\text { Proposed } \\
\text { one-phase } \\
\text { classifica- } \\
\text { tion } \\
\text { system }\end{array}$ & SRs & $\begin{array}{c}17 \text { classes of FRs and NFRs } \\
\text { (6 classes of FRs and } 11 \\
\text { classes of NFRs) }\end{array}$ & $\begin{array}{c}\text { Ensemble DL-based } \\
\text { model (BiLSTM-LSTM- } \\
\text { CNN-GRU) }\end{array}$ & PROMISE & $\begin{array}{c}\text { Accuracy: } 92.56 \% \\
\text { Precision: } 0.93 \\
\text { Recall: } 0.93 \\
\text { F1 Score: } 0.93\end{array}$ \\
\hline $\begin{array}{c}2021 \\
\text { Proposed } \\
\text { two-phase } \\
\text { classifica- } \\
\text { tion } \\
\text { system }\end{array}$ & SRs & $\begin{array}{l}\text { 1. Phase one: Binary } \\
\text { classification into FRs or } \\
\text { NFRs } \\
\text { 2. Phase two: Multi-class } \\
\text { classification of FRs (6 } \\
\text { classes) and NFRs ( } 11 \\
\text { classes) }\end{array}$ & $\begin{array}{c}\text { Ensemble DL-based } \\
\text { model (BiLSTM-LSTM- } \\
\text { CNN-GRU) }\end{array}$ & PROMISE & $\begin{array}{l}\text { 1.Accuracy: } 95.7 \% \\
\text { Precision: } 0.96 \\
\text { Recall: } 0.96 \\
\text { F1 Score: } 0.96 \\
\text { 2. Accuracy: } 93.4 \% \\
\text { Precision: } 0.94 \\
\text { Recall: } 0.93 \\
\text { F1 Score: } 0.93\end{array}$ \\
\hline
\end{tabular}

Comparing the proposed systems with the existing state-of-the-art methods proved that it improved the accuracy and outperformed the other approaches. The conducted experiments proved the success of the proposed systems using the ensemble DL approach in the binary classification of SRs into FRs or NFRs, subcategories of NFRs, and subcategories of FRs, which have received the least attention from authors previously.

\section{Conclusions and Future Work}

This study utilized ensemble DL methods to develop one- and two-phases classification systems to classify SRs in different ways: Binary and 17 multi-class classification of FRs and NFRs. The main objective was to enhance the accuracy and availability. The BiLSTM, CNN, LSTM, and GRU ensemble models were used as base DL models. Compared to a number of existing state-of-the-art ensemble methods, it was found that accuracy per class as a weight ensemble method was the best among the other ensembles. The proposed systems are considered from the few complete SR classification systems. The systems provide binary and multi-class classification with a high accuracy, as the one-phase system achieved a $92.56 \%$ accuracy, while the two-phase classification system reached $95.75 \%$ in the binary phase and $93.4 \%$ in the multi-class classification phase. Thus, it can be concluded that both of the proposed systems have leading performance results, with the two-phase classification system being better than the one-phase classification system.

The goal of this work was to introduce and provide SR classification systems that would help software engineers, developers, and analysts to produce complete SRs to build reliable software systems. These SR classification systems are based on the ensemble approach, which was applied using DL models for the first-time. These systems are complete systems, as they include binary classification, multi-class classification of FRs, and multiclass classification of NFRs, with a maximum number of classes. The accuracy is high, and its robustness was confirmed compared to the existing state-of-the-art approaches.

The limitations of the proposed model and the classification systems that are based on it include supporting one language, as it could support multiple languages. Moreover, it can be upgraded to deal with a whole document that is unstructured and can extract sentences of SRs from it instead of dealing with extracted structured sentences. 
Author Contributions: Conceptualization, F.E. and N.R.; methodology, N.R., F.E. and L.E.; software, N.R.; validation, N.R., F.E. and L.E.; formal analysis, L.E.; investigation, F.E.; resources, L.E. and N.R.; data curation, N.R.; writing-original draft preparation, N.R.; writing-review and editing, L.E.; visualization, N.R.; supervision, F.E. and L.E.; project administration, L.E.; funding acquisition, N.R. All authors have read and agreed to the published version of the manuscript.

Funding: This research received no external funding.

Data Availability Statement: Not applicable.

Conflicts of Interest: The authors declare no conflict of interest.

\section{References}

1. Tiun, S.; Mokhtar, U.A.; Bakar, S.H.; Saad, S. Classification of functional and non-functional requirement in software requirement using Word2vec and fast Text. J. Phys. Conf. Ser. 2020, 1529, 042077. [CrossRef]

2. IEEE Standard Glossary of Software Engineering Terminology. In IEEE Std 729-1983; IEEE: Manhattan, NY, USA, $1990 ;$ pp. 1-84. [CrossRef]

3. Canedo, E.D.; Mendes, B.C. Software requirements classification using machine learning algorithms. Entropy $2020,22,1057$. [CrossRef] [PubMed]

4. Alomari, R.; Elazhary, H. Implementation of a formal software requirements ambiguity prevention tool. Int. J. Adv. Comput. Sci. Appl. 2018, 9, 424-432. [CrossRef]

5. Kurtanovic, Z.; Maalej, W. Automatically Classifying Functional and Non-functional Requirements Using Supervised Machine Learning. In Proceedings of the 2017 IEEE 25th International Requirements Engineering Conference (RE), Lisbon, Portugal, 4-8 September 2017; IEEE: Piscataway, NJ, USA, 2017; pp. 490-495.

6. Del Carpio, A.F.; Angarita, L.B. Trends in Software Engineering Processes using Deep Learning: A Systematic Literature Review. In Proceedings of the 2020 46th Euromicro Conference on Software Engineering and Advanced Applications (SEAA), Portoroz, Slovenia, 26-28 August 2020; IEEE: Piscataway, NJ, USA, 2020; Volume 33, pp. 445-454.

7. Navarro-Almanza, R.; Juarez-Ramirez, R.; Licea, G. Towards Supporting Software Engineering Using Deep Learning: A Case of Software Requirements Classification. In Proceedings of the 2017 5th International Conference in Software Engineering Research and Innovation (CONISOFT), Merida, Mexico, 25-27 October 2017; IEEE: Piscataway, NJ, USA, 2017; Volume 2018, pp. 116-120.

8. Brownlee, J. Ensemble Learning Methods for Deep Learning Neural Networks. Available online: https:/ / machinelearningmastery. com/ensemble-methods-for-deep-learning-neural-networks / (accessed on 2 March 2021).

9. Ott, D. Automatic Requirement Categorization of Large Natural Language Specifications at Mercedes-Benz for Review Improvements. In Proceedings of the 19th International Conference on Requirements Engineering: Foundation for Software Quality, ser. REFSQ, Essen, Germany, 8-11 April 2013; Springer: Berlin/Heidelberg, Germany, 2013; pp. 50-64.

10. Winkler, J.; Vogelsang, A. Automatic Classification of Requirements Based on Convolutional Neural Networks. In Proceedings of the 2016 IEEE 24th International Requirements Engineering Conference Workshops (REW), Beijing, China, 12-16 September 2016; IEEE: Piscataway, NJ, USA, 2016; pp. 39-45.

11. Rahimi, N.; Eassa, F.; Elrefaei, L. An Ensemble Machine Learning Technique for Functional Requirement Classification. Symmetry 2020, 12, 1601. [CrossRef]

12. Slankas, J.; Williams, L. Automated extraction of non-functional requirements in available documentation. In Proceedings of the 2013 1st International Workshop on Natural Language Analysis in Software Engineering (NaturaLiSE), San Francisco, CA, USA, 25 May 2013; IEEE: Piscataway, NJ, USA, 2013; pp. 9-16. [CrossRef]

13. Cleland-Huang, J.; Settimi, R.; Zou, X.; Solc, P. Automated classification of non-functional requirements. Requir. Eng. 2007, 12, 103-120. [CrossRef]

14. Casamayor, A.; Godoy, D.; Campo, M. Identification of non-functional requirements in textual specifications: A semi-supervised learning approach. Inf. Softw. Technol. 2010, 52, 436-445. [CrossRef]

15. Li, L.F.; Jin-An, N.C.; Kasirun, Z.M.; Piaw, C.Y. An Empirical comparison of machine learning algorithms for classification of software requirements. Int. J. Adv. Comput. Sci. Appl. 2019, 10, 258-263. [CrossRef]

16. Casamayor, A.; Godoy, D.; Campo, M. Semi-Supervised Classification of Non-Functional Requirements: An Empirical Analysis. Intel. Artif. 2010, 13, 35-45. [CrossRef]

17. Haque, M.A.; Rahman, M.A.; Siddik, M.S. Non-Functional Requirements Classification with Feature Extraction and Machine Learning: An Empirical Study. In Proceedings of the 1st International Conference on Advances in Science, Engineering and Robotics Technology 2019, Dhaka, Bangladesh, 3-5 May 2019.

18. Fong, V. Software Requirements Classification Using Word Embeddings and Convolutional Neural Networks. Master's Thesis, California Polytechnic State University, San Luis Obispo, CA, USA, June 2018.

19. Hayes, J.; Li, W.; Rahimi, M. Weka meets TraceLab: Toward Convenient Classification: Machine Learning for Requirements Engineering Problems: A Position Paper. In Proceedings of the 2014 IEEE 1st International Workshop on Artificial Intelligence for Requirements Engineering (AIRE), Karlskrona, Sweden, 26 August 2014; IEEE: Piscataway, NJ, USA, $2014 ;$ pp. 9-12. 
20. Hey, T.; Keim, J.; Koziolek, A.; Tichy, W.F. NoRBERT: Transfer Learning for Requirements Classification. In Proceedings of the 28th IEEE International Conference on Requirements Engineering, Zurich, Switzerland, 31 August-4 September 2020.

21. Zhang, K.; Liu, N.; Yuan, X.; Guo, X.; Gao, C.; Zhao, Z.; Ma, Z. Fine-Grained Age Estimation in the Wild with Attention LSTM Networks. In IEEE Transactions on Circuits and Systems for Video Technology; IEEE: Piscataway, NJ, USA, 2019 ; pp. $3140-3152$. [CrossRef]

22. Khayyat, M.M.; Elrefaei, L.A. Manuscripts Image Retrieval Using Deep Learning Incorporating a Variety of Fusion Levels. IEEE Access 2020, 8, 136460-136486. [CrossRef]

23. Luo, L.; Yang, Z.; Lin, H.; Wang, J. Document triage for identifying protein-protein interactions affected by mutations: A neural network ensemble approach. Database 2018, 2018, bay097. [CrossRef] [PubMed]

24. Nguyen, V.Q.; Anh, T.N.; Yang, H.-J. Real-time event detection using recurrent neural network in social sensors. Int. J. Distrib. Sens. Netw. 2019, 15, 155014771985649. [CrossRef]

25. Pan, M.; Zhou, H.; Cao, J.; Liu, Y.; Hao, J.; Li, S.; Chen, C.H. Water Level Prediction Model Based on GRU and CNN. IEEE Access 2020, 8, 60090-60100. [CrossRef]

26. Shahid, F.; Zameer, A.; Muneeb, M. Predictions for COVID-19 with deep learning models of LSTM, GRU and Bi-LSTM. Chaos Solitons Fractals 2020, 140, 110212. [CrossRef] [PubMed]

27. Demir, N. Ensemble Methods: Elegant Techniques to Produce Improved Machine Learning Results. Available online: https: / / www.toptal.com/machine-learning/ensemble-methods-machine-learning (accessed on 10 October 2020).

28. Brownlee, J. What is a Confusion Matrix in Machine Learning? Available online: https:/ / machinelearningmastery.com/confusionmatrix-machine-learning/ (accessed on 1 October 2020).

29. Baccouche, A.; Garcia-Zapirain, B.; Olea, C.C.; Elmaghraby, A. Ensemble deep learning models for heart disease classification: A case study from Mexico. Information 2020, 11, 207. [CrossRef]

30. Jethwani, T. Difference Between Categorical and Sparse Categorical Cross Entropy Loss Function. Available online: https: / / leakyrelu.com/2020/01/01/difference-between-categorical-and-sparse-categorical-cross-entropy-loss-function/ (accessed on 11 November 2020).

31. Harikrishnan, N.B. Confusion Matrix, Accuracy, Precision, Recall, F1 Score. Available online: https:/ / medium.com/analyticsvidhya/confusion-matrix-accuracy-precision-recall-f1-score-ade299cf63cd (accessed on 28 May 2021).

32. Brownlee, J. Random Oversampling and Undersampling for Imbalanced Classification. Available online: https:// machinelearningmastery.com/random-oversampling-and-undersampling-for-imbalanced-classification/ (accessed on 5 June 2021). 